\title{
Perfectly Matched Layers for time-harmonic second order elliptic problems
}

\author{
A. Bermúdez • L. Hervella-Nieto • \\ A. Prieto - R. Rodríguez
}

\begin{abstract}
The main goal of this work is to give a review of the Perfectly Matched Layer (PML) technique for time-harmonic problems. Precisely, we focus our attention on problems stated in unbounded domains, which involve second order elliptic equations writing in divergence form and, in particular, on the Helmholtz equation at low frequency regime. Firstly, the PML technique is introduced by means of a simple porous model in one dimension. It is emphasized that an adequate choice of the so called complex absorbing function in the PML yields to accurate numerical results. Then, in the two-dimensional case, the PML governing equation is described for second order partial differential equations by using a smooth complex change of variables. Its mathematical analysis and some particular examples are also included. Numerical drawbacks and optimal choice of the PML absorbing function are studied in detail. In fact, theoretical and numerical analysis show the advantages of using non-integrable absorbing functions. Finally, we present some relevant real life numerical simulations where the PML technique is widely and successfully used although they are not covered by the standard theoretical framework.
\end{abstract}

Keywords Time-harmonic problems · Perfectly Matched Layers · Second order elliptic problems

PACS PACS 43.20n · PACS 02.60Lj · 02.70.Dh

A. Bermúdez

Departamento de Matemática Aplicada, Universidade de Santiago de Compostela, 15782 Santiago de Compostela, Spain. E-mail: alfredo.bermudez@usc.es

L. Hervella-Nieto

Departamento de Matemáticas, Universidade da Coruña, 15071 A Coruña, Spain. E-mail: luisher@udc.es

A. Prieto

Departamento de Matemática Aplicada, Universidade de Santiago de Compostela, 15782 Santiago de Compostela, Spain. E-mail: andres.prieto@usc.es

Applied and Computational Mathematics, 217-50 California Institute of Technology, Pasadena, CA 91125 USA. E-mail: maprieto@acm.caltech.es

R. Rodríguez

CI2MA Departamento de Ingeniería Matemática, Universidad de Concepción, Concepción, Chile. E-mail: rodolfo@ing-mat.udec.cl 


\section{Introduction}

Most numerical simulation involving mechanical models are governed by partial differential equations which can be stated either in bounded or unbounded domains depending on the physics of the problem.

If the domain is assumed to be bounded, the numerical difficulties to ensure an accurate and reliable numerical simulation are related to the use of adequate discretization methods.

However, if the domain where the model is defined is unbounded, a preliminary problem arises before the use of a discretization technique: how to apply a numerical method to a problem that, by its original nature, is not finite discretizable.

If we are only interested in computing an approximate solution in a small portion of the unbounded domain, the first naive and tentative approach could consist of truncating the unbounded domain to obtain a bounded computational one, and then to proceed as usual by applying our favorite discretization method.

For instance, as naive as it looks, due to the exponential decay of the solutions at the infinity in the Poisson or Stokes problem (see [79] and references therein), if the truncation of the unbounded domain is done far enough from the physical domain of interest, the approximate solution will be accurate and the truncation error will keep the same order of magnitude as the numerical error arising from the discretization procedure.

However, if we try to proceed analogously for the Helmholtz problem stated in an unbounded domain, a simple truncation of the domain leads to spurious numerical results. This different numerical behavior comes from the oscillatory pattern of the Helmholtz solution, which does not decay exponentially when the spatial coordinates tend to infinity.

Since our final goal is to compute an accurate numerical approximation of the solution of a physical problem by using a discrete procedure, at some stage of the algorithm we have to truncate the original unbounded domain. Thus, we need to know how to make it without perturbing too much the solution of the original problem.

It is obvious that, ideally, the method to truncate the computational domain should be accurate, efficient, easy to implement, robust to fitting parameters, and applicable for a wide class of problems.

For instance, in the framework of time-harmonic acoustics, the first step for the numerical solution of any scattering problem stated in an unbounded domain entails an inherent difficulty: to choose boundary conditions to replace the Sommerfeld radiation condition at infinity (see, for instance, [39]).

For this purpose there exist several classical numerical techniques: boundary element methods, infinite element methods, approximate Dirichlet-to-Neumann operators based on truncating Fourier expansions, absorbing boundary conditions, etc. Their potential advantages have been widely studied in the literature (see, for instance, $[5,32$, 48,63], and [39] for a classical review on this subject).

Let us make some comments on the last mentioned technique: local absorbing boundary conditions (ABCs) can be used to handle unbounded problems. Those by Bayliss and Turkel [9], Givoli [38], Engquist and Majda [33], and Feng [35] are among the most widely used. However, while computer implementation of lowest order ABCs is very easy, good accuracy is only achieved for higher order ones [72], because ABCs conditions are not fully non-reflecting on the truncated boundary of the computational domain. As a consequence, high accuracy using ABCs leads to a substantial computa- 
tional cost and increases the difficulty of implementation. Recently, a promising way has been open: higher order ABCs not involving higher order derivatives (see $[40,50]$ ). Moreover, new ABCs has been developed recently (see [41,42] and references therein) for time domain problems.

Let us remark that, if the domain of the original problem is truncated with a sphere, then the Dirichlet-to-Neumann (DtN) boundary condition is exactly known (see $[39,61]$ ). However, this boundary condition involves an infinite series which must be truncated for its numerical use. Moreover, the exact DtN condition is non local, leading to dense blocks in the linear system to be solved when a finite element method is used.

Another alternative approach to make the truncation of unbounded domains is the so-called Perfectly Matched Layer (PML) method, which was introduced by Berenger [1315] for electromagnetic problems. It is based on introducing an absorbing layer of anisotropic damping material surrounding the domain of interest. This has the effect of a thin sponge which absorbs the scattered field radiated to the exterior of this domain. This layer is said as 'perfectly matched' because its interface with the physical domain does not produce spurious reflections inside the domain of interest, as it is the case of ABCs.

This method has been applied to different problems. It was initially settled for Maxwell's equations in electromagnetism $[11,13]$ and subsequently used for the scalar Helmholtz equation $[44,67,78]$, advective acoustics [2,10,47], shallow water waves [62], elasticity [8,29], poroelastic media [81], and other hyperbolic problems (see for instance [59] among many other papers).

Instead of restricting our attention to a unique problem, the introduction of the PML governing equation can be unified for several time-harmonic problems. Hence, in the present paper we first deal with the description and the partially incomplete mathematical analysis of the PML technique for second order elliptic problems. Then, some sections are devoted to the optimal fitting of this technique for time-harmonic scattering problems in linear acoustics, i.e., for the scalar Helmholtz equation. In both cases, for the sake of simplicity, we restrict ourselves to the two-dimensional case.

Although this work is entirely devoted to time-harmonic problems, let us say a few words about time dependent problems. The PML technique was originally developed for being applied in the framework of the finite difference time domain (FDTD) methods.

In deducing the PML technique [13], Berenger used an artificial splitting of the physical unknowns, to force the tangential components of both velocities (in the acoustic medium and in the PML layer) to coincide on the interface for any frequency and any angle of incidence, thus guaranteeing the absence of spurious reflections [48]. However, from a mathematical point of view, this non physical splitting has been shown unnecessary to state the PML equations.

In fact, some authors (see for instance [1]), based on the fact that the PML model is a zero order perturbation of a weakly hyperbolic first order system (see [54]), assumed that the Berenger PML model could be ill-posed for time dependent problems, despite of its good numerical accuracy. Now it is well known that this is not the case.

Notice that, the well posedness of the time domain PML formulations does not depend on the absorbing behavior of layer. In fact, as shown in [11], their weak stability only relies on the symbol expression of the hyperbolic differential operator.

Obviously, it is straightforward to introduce the time-harmonic PML technique by means of the time-Fourier transform, from the classical Berenger's split formulation. However, being incoherent with the historic development of the PML technique, we 
introduce the time-harmonic version of the Berenger's split formulation in Section 2 as a modification of the Darcy's like porous model.

There exist a huge amount of possible alternatives to introduce a PML technique in time dependent problems. Let us mention, for instance, the split-field PML (original derived by Berenger); the unaxial PML (UPML) which can also be understood as an artificial anisotropic material (see $[71,36,37]$ ); the complex shifted PML (see [55]) which generalizes the positive profile of the absorbing functions and allows working with arbitrary complex absorbing functions; the Zhao-Cangellaris formulation (see [82]) or, equivalently, formulations with auxiliary differential equations (ADE) which recover formally the equations of the original hyperbolic problem; and the convolutional PML (see [69]) that introduce the absorbing coefficients of the PML by means of convolutional products in the time variable.

Contrary to this wide variety of choices for time dependent problems, most of them can be reduced to a unique PML governing equation in the frequency domain, eventually with different absorbing functions. The exception arises from the UPML technique whose governing equation can be understood as the adjoint formulation of Berenger equations (see [79] for details).

Let us remark that, even if the governing equation is the same in the time-harmonic regime, this fact does not imply that the solutions obtained with two different PML formulations remain equal for time dependent problems. For instance, the solutions of the Berenger's split and the Zhao-Cangellaris formulation are different in the time domain. Nevertheless, both model are equivalent, because the solution of each of them can be written in terms of the solution of the other (see [11]).

Moreover, Chew and Weedon [25] and Rappaport [68] showed that the PML equations can be obtained using a complex-valued coordinate stretching, in the framework of time-harmonic wave propagation. Related to this, Lassas et al. $[58,56]$ showed that the PML, and in general a family of absorbing conditions, can be obtained by using complex Riemann metric tensors.

The use of the complex stretching of coordinates is closely related to an old technique called complex scaling technique (also referenced as analytic dilatation technique), which was developed in the seventies of the last century to simulate numerically the eigen-states of quantum and atomic systems (see the first contributions [3,73] or the textbook [45] and references therein). In fact, part of the numerical behavior of the PML technique was already described in these pioneer works.

Furthermore, in spite of the fact that the PML has been originally settled in Cartesian coordinates by Berenger, Collino and Monk [28] proposed a similar complex-valued change of coordinates to build a PML in curvilinear coordinates, as we have also emphasized in [19].

In practice, since the PML has to be truncated at a finite distance of the physical domain of interest, its exterior boundary produces artificial reflections. Theoretically, these reflections are of minor importance because of the exponential decay of the acoustic waves inside the PML. In fact, for Helmholtz-type scattering problems, Lassas and Somersalo [57] proved, by using boundary integral equation techniques, that the approximate solution obtained by the PML method converges exponentially to the exact solution in the computational domain as the thickness of the layer tends to infinity. This result was generalized by Hohage et al. [46] using techniques based on the pole condition. Moreover, Bécache et al. [10] proved an analogous result for the convected Helmholtz equation. 
When the problem is discretized to be numerically solved, the approximation error typically becomes larger. Increasing the thickness of the PML may be a remedy, but it is not always possible because of the computational cost. An alternative is to take larger values for the absorbing function involved in the complex-valued coordinate stretching. However, Collino and Monk [27] showed that this methodology may produce an error growth in the discretized problem. Consequently, an optimization problem arises: given a data set and a mesh, to choose the optimal absorbing function to minimize the error.

In this framework, Asvadurov et al. [6] proposed a pure imaginary stretching to optimize the PML error. They recovered exponential error estimates using finite-difference grid optimization. However, to the best of our knowledge, the optimization problem is still open in that there is no optimal criterion to choose a bounded absorbing function independently of data and meshes. As an alternative procedure to avoid this drawback, our main contribution (see $[16,19,17]$ ) is based on using an absorbing function with unbounded integral on the PML.

The outline of the paper is as follows. Firstly, in Section 2 we introduce a sponge layer model to truncate the unbounded computational domain and, as an improvement of this model, the PML governing equation is derived modifying the stiffness term of the Darcy's like porous model. A detailed comparison between the Darcy's like porous model and the PML is done by solving one-dimensional acoustic problems.

Then, in Section 3, after the introduction of the so called absorbing function for the PML, we illustrate the role that the real and imaginary parts of the absorbing function play with respect to the behavior of the PML solution in three different onedimensional problems. In this simple framework, we include a straightforward result ensuring the exponential decay of the root mean square error between the solution of the original problem, stated in an unbounded domain, and the solution computed with the PML technique.

Following the ideas developed for the analytic dilatation technique, the PML governing equation is derived for second order problems written in divergence form in Section 4. For the sake of simplicity we restrict ourselves to two-dimensional problems and show that the derived PML model recovers the well-known Cartesian and radial PML equations for isotropic acoustic fluids and linear elastic materials.

In Section 5 we describe a theoretical result which guarantees the existence and uniqueness of solution for the PML problem when second order elliptic equations are involved. For the sake of completeness, the sketch of the proof is also included. Since this theoretical result is not totally satisfactory, a particular and more complete result for the Helmholtz equation is also reported from the work by Bramble and Pasciak [22], where not only the uniqueness of solution but also the exponential decay of the truncation error are proved.

As a preliminary study, in Section 6 we include the analysis of the plane wave propagation with oblique incidence in a two-dimensional unbounded domain. We show that a PML method based on a non-integrable absorbing function allows recovering the exact solution in the domain of interest. Section 6 also includes some numerical results which illustrate the efficiency and accuracy of the PML technique with a nonintegrable absorbing function. A comparison using different absorbing profiles is also given.

Moreover, we review the theoretical results guaranteeing the accuracy of the radial PML technique with non-integrable absorbing functions. In fact, we show the exactness of the PML technique when a non-integrable smooth absorbing function is used in the 
construction of the perfectly matched layer. The numerical accuracy of this problem is also illustrated with an academic problem where the exact solution is known.

Three relevant real-life numerical simulations, which are not supported by any theoretical result until now, are presented in Section 7. Despite of the theoretical lack for time-harmonic problems involving half-space domains, linear elastic waveguides or stratified acoustic fluids, the accuracy of the numerical results is usually assumed in the literature.

Finally, some remarks about further research on the PML technique for timeharmonic problems, the PML optimal parameter fitting and other related open problems are discussed in Section 8.

\section{A mechanical derivation for a simple PML model}

If we focus our attention on real life engineering solutions, which are applied to isolate a physical region of interest without introducing spurious reflections (as in the case of anechoic rooms), we observe that most of them are based on sponge layers (see [12]). Thus, it is quite natural to consider porous layers for our computational purpose.

To illustrate the numerical performance of this strategy, we are going to solve a simple one-dimensional toy problem whose solution is a plane wave with amplitude one and null phase, propagating in the positive real axis. More precisely, we consider the following problem:

Given $\omega>0$, find the scalar field $u$ such that

$$
\begin{aligned}
-\left(\rho c^{2} u^{\prime}\right)^{\prime}-\omega^{2} \rho u & =0 & & \text { in }(0,+\infty), \\
u & =1 & & \text { at } x=0, \\
\rho c u^{\prime}-i \omega \rho u & =0 & & \text { at } x \rightarrow+\infty,
\end{aligned}
$$

where $u$ represents the displacement field, $c$ is the sound speed, $\rho$ the mass density, and $\omega$ the angular frequency. If all the physical parameters are constant then the solution is given by $u(x)=e^{i(\omega / c) x}$.

As we have described above, we truncate the interval $(0,+\infty)$ with an absorbing porous layer of finite thickness. With this purpose, we introduce the most simple model for porous materials, which can be derived from the Darcy's law for incompressible fluid flows (see [4]).

The physical domain of interest is fixed to be $(0,1)$ and the porous layer is placed in $(1,1+L)$ with $L>0$. This truncated problem is written in terms of the coupled displacements $\left(u_{\mathrm{F}}, u_{\mathrm{A}}\right)$, where $u_{\mathrm{F}}$ is the solution computed in the physical domain and $u_{\mathrm{A}}$ is the solution in the porous layer. The equations describing the coupled problem 
are

$$
\begin{aligned}
-\left(\rho c^{2} u_{\mathrm{F}}^{\prime}\right)^{\prime}-\omega^{2} \rho u_{\mathrm{F}}=0 & \text { in }(0,1), \\
-\left(\frac{\rho c^{2}}{\phi \mu} u_{\mathrm{A}}^{\prime}\right)^{\prime}-\omega^{2} \rho\left(1+i \frac{\sigma}{\omega \rho}\right) u_{\mathrm{A}}=0 & \text { in }(1,1+L), \\
u=1 & \text { at } x=0, \\
u_{\mathrm{F}}=u_{\mathrm{A}} & \text { at } x=1, \\
\rho c^{2} u_{\mathrm{F}}^{\prime}=\frac{\rho c^{2}}{\phi \mu} u_{\mathrm{A}}^{\prime} & \text { at } x=1, \\
u_{\mathrm{A}}=0 & \text { at } x=1+L,
\end{aligned}
$$

where $\sigma$ is the flow resistivity of the porous layer, $\phi$ is the porosity and $\mu$ is the ratio of specific heats of fluid.

Here, and through the rest of the paper, we consider the branch of the square root with positive real part. If we introduce the wavenumbers $k_{\mathrm{F}}(\omega)=\omega / c, k_{\mathrm{A}}(\omega)=$ $(\omega / c) \sqrt{\phi \mu(1+i \sigma /(\omega \rho))}$ and the characteristic impedances $Z_{\mathrm{F}}(\omega)=\rho c, Z_{\mathrm{A}}(\omega)=$ $\rho c \sqrt{\phi \mu(1+i \sigma /(\omega \rho))}$ of both media, and assume that all the physical parameters are constant, a direct computation leads to

$$
\begin{array}{lr}
u_{\mathrm{F}}(x)=e^{i k_{\mathrm{F}} x}+A\left(-e^{i k_{\mathrm{F}}(x+1)}+e^{-i k_{\mathrm{F}}(x-1)}\right) & \text { in }(0,1), \\
u_{\mathrm{A}}(x)=B\left(-e^{i k_{\mathrm{A}}(x-(1+2 L))}+e^{-i k_{\mathrm{A}}(x-1)}\right) & \text { in }(1,1+L),
\end{array}
$$

where

$$
\begin{aligned}
A & =\frac{Z_{\mathrm{F}} \sin \left(k_{\mathrm{A}} L\right)-i Z_{\mathrm{A}} \cos \left(k_{\mathrm{A}} L\right)}{2\left(Z_{\mathrm{F}} \sin \left(k_{\mathrm{A}} L\right) \cos \left(k_{\mathrm{F}}\right)+Z_{\mathrm{A}} \sin \left(k_{\mathrm{F}}\right) \cos \left(k_{\mathrm{A}} L\right)\right)}, \\
B & =\frac{Z_{\mathrm{F}} e^{i k_{\mathrm{A}} L}}{2\left(Z_{\mathrm{F}} \sin \left(k_{\mathrm{A}} L\right) \cos \left(k_{\mathrm{F}}\right)+Z_{\mathrm{A}} \sin \left(k_{\mathrm{F}}\right) \cos \left(k_{\mathrm{A}} L\right)\right)} .
\end{aligned}
$$

If we set the physical parameters to $\rho=1.0 \mathrm{~kg} / \mathrm{m}^{3}, c=340.0 \mathrm{~m} / \mathrm{s}, \omega=6800 \mathrm{rad} / \mathrm{s}$, $\sigma=3500 \mathrm{~kg} /\left(\mathrm{m}^{3} \mathrm{~s}\right), \phi=0.71, \mu=1.4$, and $L=1.0 \mathrm{~m}$, the plots in Figure 1 show the real part of the solution $u$ for the original unbounded problem (1)-(3) and the real part of the solution $\left(u_{\mathrm{F}}, u_{\mathrm{A}}\right)$ for the coupled problem (4)-(9).

With this physical setting, the $\mathrm{L}^{2}$-relative error in the physical domain $(0,1.0)$, i.e, the quantity $\left(\int_{0}^{1}\left|u(x)-u_{\mathrm{F}}(x)\right|^{2} \mathrm{~d} x\right)^{\frac{1}{2}} /\left(\int_{0}^{1}|u(x)|^{2} \mathrm{~d} x\right)^{\frac{1}{2}}$, is equal to $15.5 \%$. In fact, a simple inspection of the formulas above allows us to verify that the difference between $u$ and $u_{\mathrm{F}}$ comes from the second term in (10), which corresponds to a reflected plane wave in the physical domain.

Hence, to approximate accurately $u$ by $u_{\mathrm{F}}$, the reflected amplitude should be null and the incident amplitude should remain equal to one. Obviously, we could argue that the flow resistivity or the thickness of the porous layer has not been chosen adequately. In Figure 2, we show the $\mathrm{L}^{2}$-relative error for a fixed flow resistivity, varying the thickness of the layer, and also for a fixed thickness varying the flow resistivity.

In both cases we check that the relative error is not smaller than around $10 \%$ and this error does not decrease if the thickness of the layer or the flow resistivity are increased. On the contrary, if $\sigma$ is fixed then the relative error tends to be constant for large thickness and, if we fix $L$, the error increases monotonically for large values of $\sigma$. 

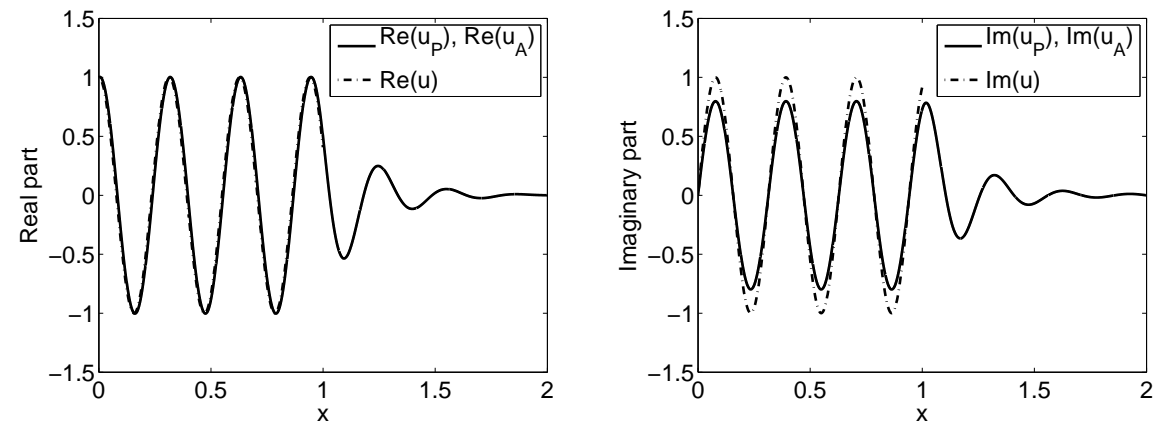

Fig. 1 Real and imaginary parts of the solution for problems (1)-(3) and (4)-(9).
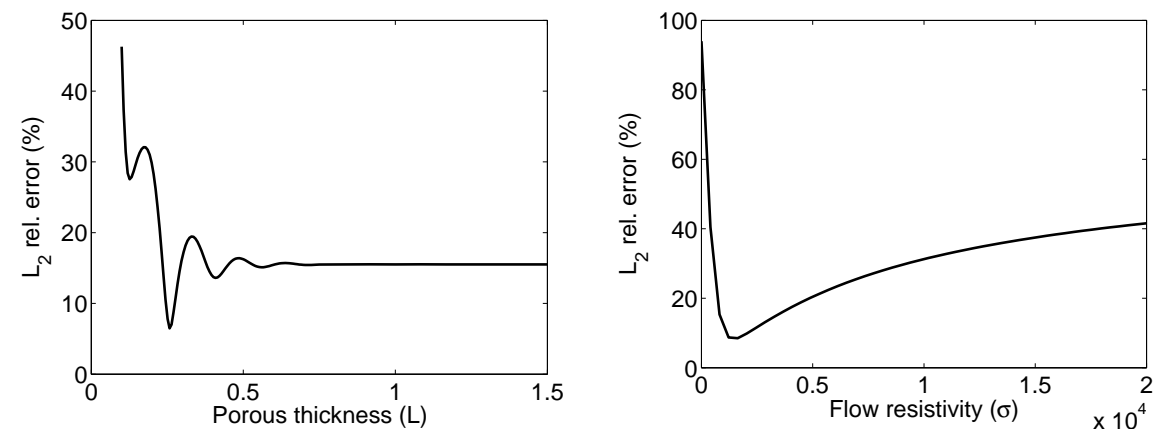

Fig. $2 \mathrm{~L}^{2}$-relative error for $\sigma=3500 \mathrm{~kg} /\left(\mathrm{m}^{3} \mathrm{~s}\right)$ varying the thickness of the porous layer (left) and for $L=1.0 \mathrm{~m}$ varying the flow resistivity (right).

In fact, both plots illustrate the same phenomenon: if the thickness of the porous layer or the value of the flow resistivity is increased then the second term in the numerator of (11) becomes null, what corresponds to the amplitude of the reflected plane wave in the porous layer. However, the second term in (10) remains far from negligible. In fact, since the real and imaginary part of $k_{\mathrm{A}}$ are positive, we have that $\tan \left(k_{\mathrm{A}} L\right)$ goes to $i$ when $L$ tends to $\infty$ and so the amplitude $A$ is proportional to $Z_{\mathrm{F}}-Z_{\mathrm{A}}$.

On one hand, if the characteristic impedances of both media do not coincide (see [23]) even for large thickness of the porous layer, the reflected plane wave generated on the interface between the physical domain and the absorbing layer is not small and the solutions $u$ and $u_{\mathrm{F}}$ remain different.

On the other hand, if $\sigma$ is small enough and $\mu \phi$ is close to one then $Z_{\mathrm{F}}$ and $Z_{\mathrm{A}}$ will be similar. Thus, $Z_{\mathrm{A}}-Z_{\mathrm{F}}$ is small and so $A$ will also become small. Consequently, the difference between $u_{\mathrm{F}}$ and $u$ will be negligible.

In conclusion, it would be convenient to make as small as possible the reflections from the interface between both media. In order to do that, there exists at least two possible solutions. On one hand, we could change the constant flow resistivity by using a variable profile in such a way that the transition between both media becomes smooth. 
On the other hand, we could think up a different governing equation for the absorbing layer, which does not generate reflections on the interface.

In the first alternative, the reflections from the porous medium can be done arbitrarily small as much as the gradient of $\sigma$ is sufficiently small (see [49] or [64]). This behavior is claimed to be based on adiabatic results which guarantee the energy dissipation in the porous layer without spurious reflections.

Precisely, this strategy consists in changing the constant flow resistivity in the absorbing layer $(1,1+L)$ by using a smooth profile, for instance, $\sigma(x)=\sigma_{0}(x-1)^{2}$ being $\sigma_{0}$ a constant value to be fitted. Now, since the flow resistivity has a variable profile, formulas (10)-(11) are not longer valid so it is not possible to write the solution in terms of reflected and transmitted plane waves. Throughout the rest of the section, a standard finite element method on a fine enough mesh has been used to compute an accurate solution for the problems involving a variable profile.

Obviously, now $\sigma(x)$ can be smoothly extended to the full domain $(0, L+1)$ as it is null at the interface $x=1$. Analogous to the analysis made with the constant profile, we have computed the $\mathrm{L}^{2}$-relative error against the thickness of the porous layer and the parameter $\sigma_{0}$. The results are shown in Figure 3.
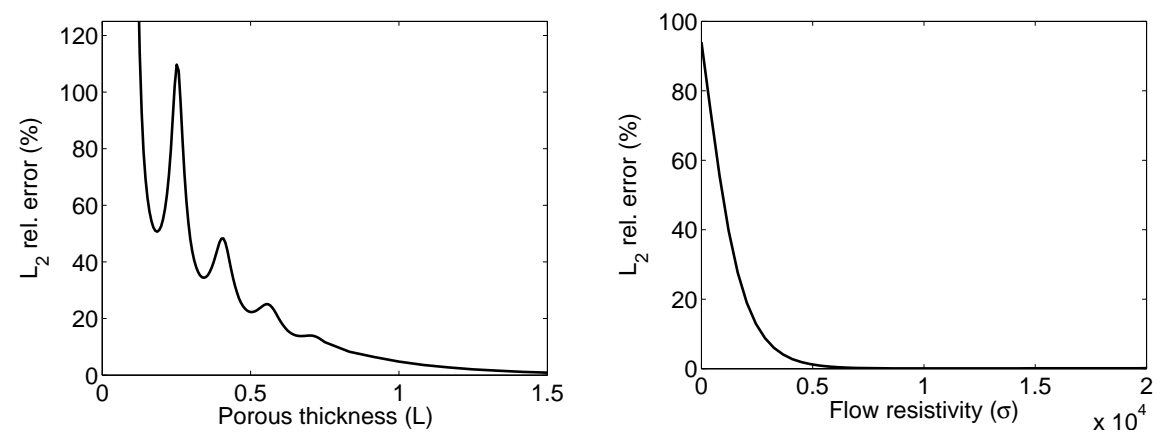

Fig. $3 \mathrm{~L}^{2}$-relative error for $\sigma_{0}=3500 \mathrm{~kg} /\left(\mathrm{m}^{3} \mathrm{~s}\right)$ varying the thickness of the porous layer (left) and for $L=1.0 \mathrm{~m}$ varying parameter $\sigma_{0}$ (right), in both cases using the smooth profile $\sigma(x)=\sigma_{0}(x-1)^{2}$.

Now, the $\mathrm{L}^{2}$-relative error is lower than $3.5 \%$ for $\sigma_{0}=3500 \mathrm{~kg} /\left(\mathrm{m}^{3} \mathrm{~s}\right)$ and $L=1.0 \mathrm{~m}$. Moreover, if the flow resistivity parameter $\sigma_{0}$ and the thickness $L$ are increased in a reasonable interval, the relative error remains small and lower than $0.5 \%$.

Thus, if the variable smooth profile for the flow resistivity is used and adequate values for $L$ and $\sigma_{0}$ are fitted, we recover accurately the solution of the original problem $u$ by computing the solution $u_{\mathrm{F}}$. In fact, some authors use this kind of slow varying smooth profiles for $\sigma$, that leads to the minimization of the energy reflected towards the physical domain from the absorbing layer (see [49] for details).

However, the procedure described above has an important drawback related with the computational cost of this strategy. Even in this simple toy problem, if we compare the thickness of the porous layer with the characteristic wavelength $\lambda$ of the problem in the physical domain, where $\lambda=2 \pi / k_{\mathrm{F}}$, we check that it has been necessary to use a thickness $L>3 \lambda$ to obtain a relative error lower than $3.5 \%$. 
In order to illustrate the behavior of the $\mathrm{L}^{2}$-relative error with respect to the wavelength, it has been depicted in Figure 4, where constant and variable profiles for $\sigma$ have been considered as above. In both cases we have fixed $\sigma_{0}=3500 \mathrm{~kg} /\left(\mathrm{m}^{3} \mathrm{~s}\right)$ and $L=1.0 \mathrm{~m}$.
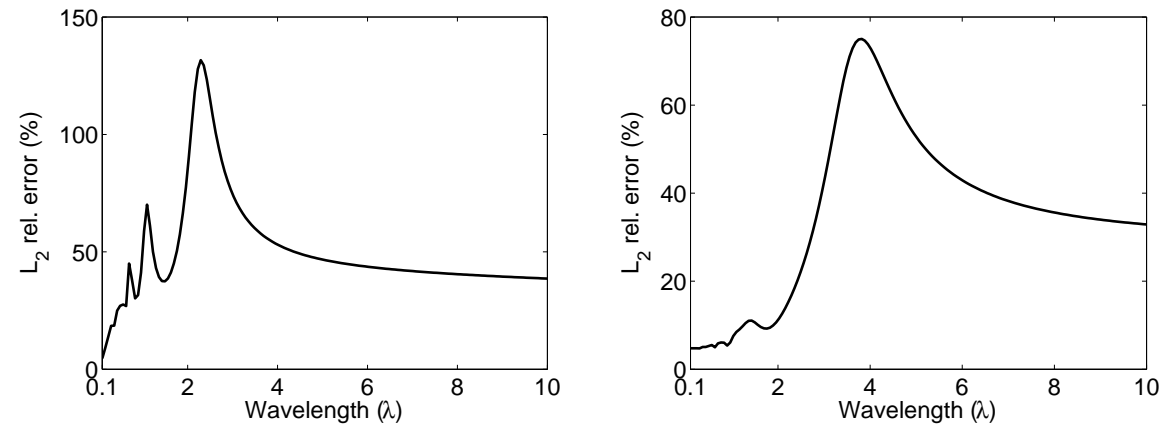

Fig. $4 \mathrm{~L}^{2}$-relative error for $\sigma_{0}=3500 \mathrm{~kg} /\left(\mathrm{m}^{3} \mathrm{~s}\right)$ and $L=1.0 \mathrm{~m}$ varying the wavelength of the problem using a constant profile $\sigma(x)=\sigma_{0}$ (left) and a variable profile $\sigma(x)=\sigma(x-1)^{2}$.

Although the relative error remains below 3\%, for small wavelengths (at high frequency regime), the truncation of the unbounded physical domain with a porous layer of finite thickness fails to approximate the original solution $u$ by using $u_{\mathrm{F}}$, for large wavelengths (at low frequency regime). This behavior is similar for the constant and for the smooth variable profiles of $\sigma$. Hence, in order to guarantee accurate results at low frequency regime it is necessary to take into account a large thickness parameter. Consequently, the strategy becomes highly expensive from a computational point of view.

In conclusion, the first of the two alternatives that we have previously announced does not achieve the purpose of elaborating an algorithm allowing us to compute accurately the solution of problem (4)-(9) with a low computational cost.

Next we explore the second alternative that was sketched above. As we have already analyzed, the main problem about the truncation of the unbounded domain $(0,+\infty)$ with a porous layer relies on the different characteristic impedances of both media. This difference produces reflected waves on the interface between the physical domain and the porous layer, what leads to inaccurate results. Hence, we are going to modify the Darcy's like porous model with the aim of satisfying the following two properties:

1. the model should have a dissipative behavior like a porous material,

2. its characteristic impedance would match the characteristic physical impedance of the media at the interface.

In the sequel, these are the conditions we are going to require to the PML model.

To verify this two properties, an additional artificial dissipation term is included in the stiffness term of the Darcy's like porous model. Consequently, the one-dimensional PML governing equation can be understood as an artificial absorbing material. More precisely, the porous bulk modulus $\left(\rho c^{2}\right) /(\phi \mu)$ is multiplied by the non-dimensional factor $\phi \mu /(1+i \sigma /(\omega \rho))$. Notice that the denominator of this expression is just the dissipative mass term multiplying the mass density in the Darcy's like model. 
Hence, we replace problem (4)-(9) by the following one:

$$
\begin{aligned}
-\left(\rho c^{2} u_{\mathrm{F}}^{\prime}\right)^{\prime}-\omega^{2} \rho u_{\mathrm{F}}=0 & \text { in }(0,1), \\
-\left(\frac{\rho c^{2}}{1+i \frac{\sigma}{\omega \rho}} u_{\mathrm{A}}^{\prime}\right)^{\prime}-\omega^{2} \rho\left(1+i \frac{\sigma}{\omega \rho}\right) u_{\mathrm{A}}=0 & \text { in }(1,1+L), \\
u=1 & \text { at } x=0, \\
u_{\mathrm{F}}=u_{\mathrm{A}} & \text { at } x=1, \\
\rho c^{2} u_{\mathrm{F}}^{\prime}=\frac{\rho c^{2}}{1+i \frac{\sigma}{\omega \rho}} u_{\mathrm{A}}^{\prime} & \text { at } x=1, \\
u_{\mathrm{A}}=0 & \text { at } x=1+L .
\end{aligned}
$$

Now, if $\sigma$ is constant, the exact coupled solution has a closed form given by

$$
\begin{array}{lr}
u_{\mathrm{F}}(x)=R e^{i k_{\mathrm{F}}(x-1)}+T e^{-i k_{\mathrm{F}}(x-1)} & \text { in }(0,1), \\
u_{\mathrm{A}}(x)=R e^{i k_{\mathrm{A}}(x-1)}+T e^{-i k_{\mathrm{A}}(x-1)} & \text { in }(1,1+L),
\end{array}
$$

where the reflected and transmitted amplitudes are respectively

$$
R=\frac{1}{e^{i k_{\mathrm{F}}}-e^{-i k_{\mathrm{F}}} e^{-2 i k_{\mathrm{A}} L}}, \quad T=-\frac{e^{-2 i k_{\mathrm{A}} L}}{e^{i k_{\mathrm{F}}}-e^{-i k_{\mathrm{F}}} e^{-2 i k_{\mathrm{A}} L}},
$$

the wavenumber of the PML model is given by $k_{\mathrm{A}}(\omega)=(\omega / c)(1+i \sigma /(\omega \rho))$, and its characteristic impedance by $Z_{\mathrm{A}}(\omega)=\rho c$.

Since we have forced the values of the characteristic impedances of both media to be equal, no reflected waves are generated at the interface between the physical domain and the absorbing one. Consequently, a reduction of the relative error between $u$ and $u_{\mathrm{F}}$ is expected. It is easy to check that the amplitudes of the coupled solution (18)-(19) can be computed from those in (10)-(11), by taking into account that $Z_{\mathrm{F}}(\omega)=Z_{\mathrm{A}}(\omega)$.

Obviously, we are now in a position to use the constant or the variable profile for parameter $\sigma$ which has lost its proper physical meaning. The solution of the coupled problem (12)-(17) has been computed with $\sigma=\sigma_{0}$ and with the variable profile $\sigma=\sigma_{0}(x-1)^{2}$ using the physical parameters described above. The real part of both solutions have been plotted in Figure 5. Now, the relative errors are much smaller than those corresponding to the truncation with the porous layer given before; precisely, they are $0.002 \%$ and $0.446 \%$ for the constant and variable cases, respectively.

In spite of improving the $\mathrm{L}^{2}$-relative errors from those obtained for the truncation strategy with a Darcy's like porous layer, the error is not negligible. In fact, this error is still produced by the spurious reflections that comes from the exterior boundary of the PML domain. Obviously, these reflections are related to the finite thickness of the absorbing layer.

Most of the literature about the PML technique in the time-harmonic regime tries to obtain an estimate of these spurious reflections in terms of the PML thickness and, somehow, in terms of the value of $\sigma_{0}$. Some of these results are reviewed in Section 5 .

Finally, to compare the behavior of the PML model with the Darcy's like model, in the plots of Figure 6 we show a similar study to previously done by analyzing the behavior of the $\mathrm{L}^{2}$-relative error versus the flow resistivity $\sigma_{0}$, the thickness of the absorbing layer $L$ and the wavelength $\lambda$. 

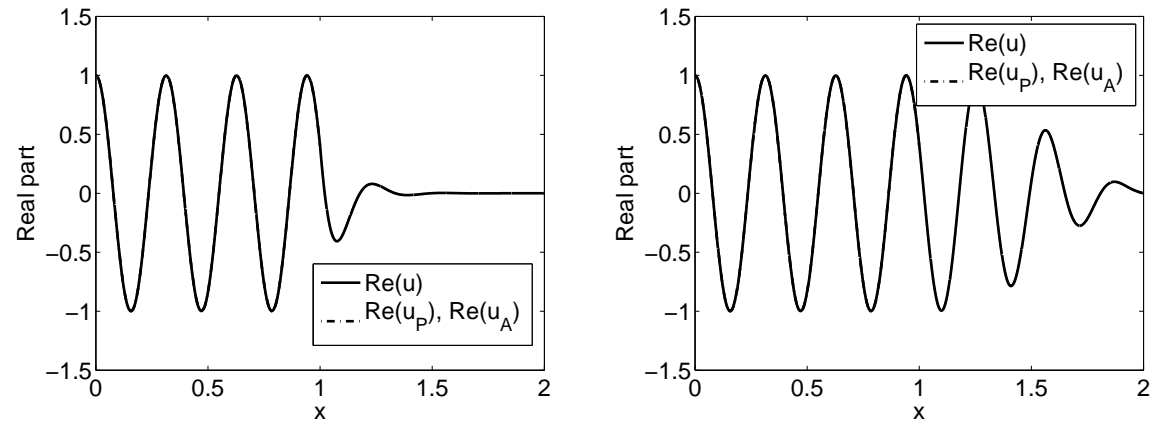

Fig. 5 Real part of the solution of problem (12)-(17) with constant profile $\sigma=\sigma_{0}$ (left) and variable profile $\sigma=\sigma_{0}(x-1)^{2}$ (right).
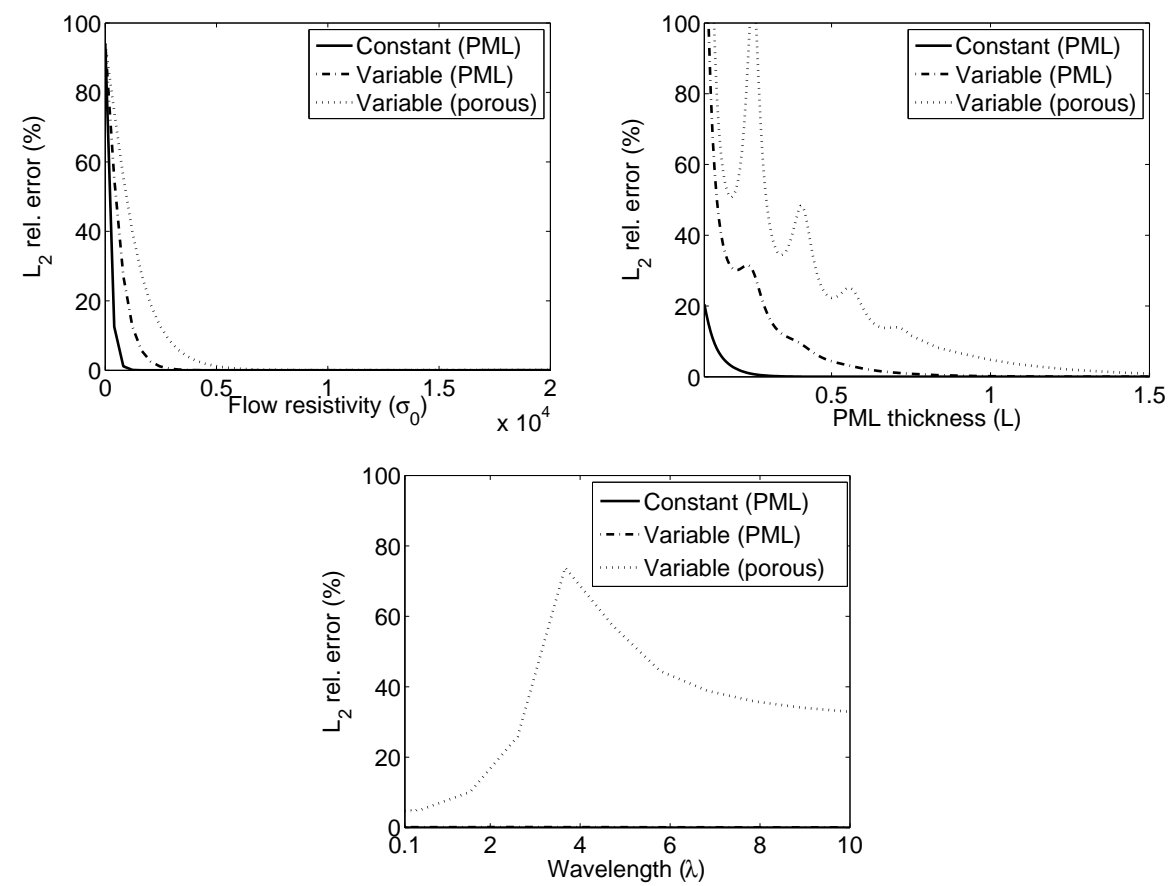

Fig. $6 \mathrm{~L}^{2}$-relative error for constant and variable profile versus the flow resistivity (upper left), the thickness of the layer (upper right), and the wavelength (lower center).

It is checked that the behavior of the relative error, when the thickness or the parameter $\sigma_{0}$ is varying, is similar to those shown in Figure 3 for the Darcy's like model with smooth variable profile for $\sigma$. However, the main difference lies in the plot of the relative error versus the wavelength. Instead of having a monotonically increasing behavior as depicted in Figure 4, the error remains constant for the considered entire range of wavelengths. This fact represents a fundamental advantage for compu- 
tational purposes, since the PML technique guarantees that the truncation error is not increasing in the low frequency regime.

\section{Absorbing behavior of PML models}

In the previous section, we have introduced the PML governing equation from a Darcy's like porous model. In fact, we have been guided by the physical meaning of the flow resistivity, which plays a main role in the dissipative behavior of PML and porous layers. However, we can suspect that this situation is fairly particular and strictly related to the one-dimensional problem considered in Section 2, where all the physical coefficients were positive. Thus, it could be claimed that the derived PML equation is only valid for the acoustic model.

The aim of this section consists of making the one-dimensional mechanical derivation of the PML governing equation developed in the previous section as general as possible. With this purpose, firstly let us recall again the two requirements stated by the PML model: 1.- dissipative behavior similar to that of porous media and 2.matching its characteristic impedance with the one of the physical medium. As we have already seen, the second item is satisfied for any value of $\sigma$. More precisely, since the characteristic impedance is defined as the bulk modulus multiplied by the factor $k_{\mathrm{A}} / \omega$, we have

$$
Z_{\mathrm{A}}(\omega)=\frac{\rho c^{2}}{1+i \frac{\sigma}{\omega \rho}} \frac{k_{\mathrm{A}}}{\omega}=\frac{\rho c^{2}}{1+i \frac{\sigma}{\omega \rho}} \frac{\omega}{c} \frac{1+i \frac{\sigma}{\omega \rho}}{\omega}=\rho c=Z_{\mathrm{F}}(\omega) .
$$

Hence, we have to pay attention only to verify the first property.

From the expression above, we must realize that the main role is not played by the parameter $\sigma$ but by the quantity $1+i \sigma /(\omega \rho)$. Following this idea, if we introduce the complex-valued function $\gamma=1+i \sigma /(\omega \rho)$ then the PML governing equation in problem (12)-(17) is written in a simpler form,

$$
-\left(\frac{\rho c^{2}}{\gamma} u_{\mathrm{A}}^{\prime}\right)^{\prime}-\omega^{2} \rho \gamma u_{\mathrm{A}}=0 \quad \text { in }(L, L+1) .
$$

Through the rest of the paper, both for one-dimensional and two-dimensional problems, the complex-valued function $\gamma$ will be called PML absorbing function. Let us remark that in the literature, other terminology as "absorption coefficient" or "damping function" can be used for $\gamma$ or, even, the term "absorbing function" can denote other different functions related with but not equal to $\gamma$.

Obviously, we could use the physical guidance given in the previous section and choose $\gamma=1+i \sigma /(\omega \rho)$ with a positive-valued function $\sigma$. However, in the case of the simple one-dimensional problem (1)-(3), the two properties of the PML model are satisfied for any complex-valued function $\gamma$, eventually being independent of $\omega$, with the unique requirement of satisfying $\operatorname{Im}(\gamma)>0$ (see Lemma 1).

Now, we are in a position to test the generality of the PML governing equation (20) in other one-dimensional problems different from (1)-(3). More specifically, to check whether (20) remains valid for other mechanical models and, moreover, whether the requirement $\operatorname{Im}(\gamma)>0$ is a sufficient condition to construct a PML governing equation that allows computing accurately the solution of general problems involving unbounded domains. 
In the rest of this section we will show that the answer to the two questions above are negative and, to illustrate it, we consider below three different one-dimensional problems where the PML technique is used. In these three problems, as it has been done previously, the unbounded domain is truncated with a PML placed in $(1, L+1)$ and the same physical parameters set in Section 2 are used, where it applies. Moreover, let us remark that two different complex absorbing functions, $\gamma=1+i \sigma_{0} / \omega$ and $\gamma=\exp (i \pi / 6)$, has been considered in these three test problems.

1. The propagation of plane pressure waves (see [23]) with an oblique angle of incidence $\theta(\theta=0$ for normal incidence) in an isotropic compressible fluid occupying the two-dimensional half-space $(0,+\infty) \times \mathbb{R}$ is described by $\exp \left(i(\omega \cos (\theta) / c) x_{2}\right) u\left(x_{1}\right)$, where $\theta \in(0, \pi / 2)$ and $u$ is the solution of the following problem:

$$
\begin{aligned}
-\left(\rho c^{2} u^{\prime}\right)^{\prime}-\omega^{2} \cos ^{2}(\theta) \rho u & =0 & & \text { in }(0,+\infty), \\
u & =1 & & \text { at } x=0, \\
\rho c u^{\prime}-i \omega \cos (\theta) \rho u & =0 & & \text { at } x \rightarrow+\infty .
\end{aligned}
$$

The $\mathrm{L}^{2}$-relative error between the exact solution of problem (21)-(23) and the one computed with the PML technique is depicted in Figure 7.
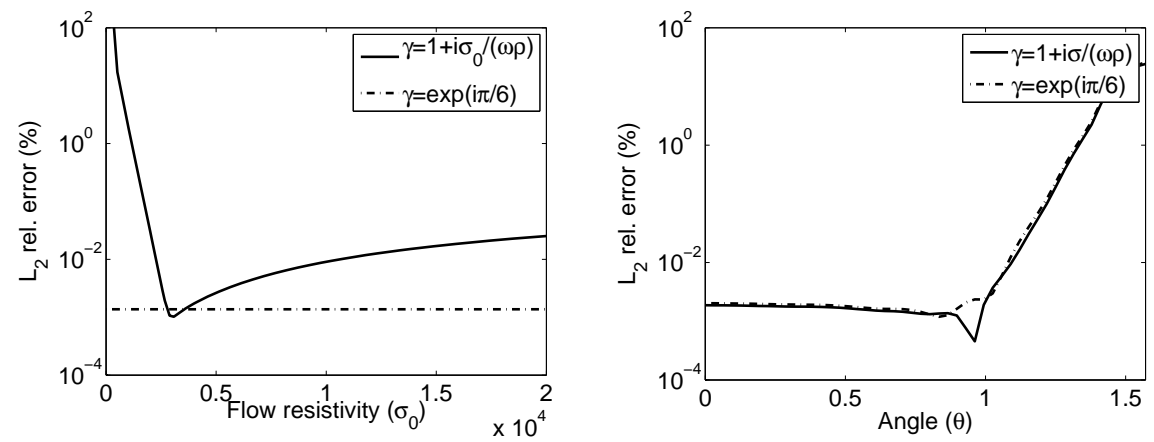

Fig. $7 \mathrm{~L}^{2}$-relative error for problem (21)-(23) varying parameter $\sigma_{0}$ with fixed $\theta=\pi / 4$ (left) and varying the angle of incidence $\theta$ with fixed $\sigma_{0}=3500 \mathrm{~kg} /\left(\mathrm{m}^{3} \mathrm{~s}\right)$.

Obviously, the truncation error for $\gamma=\exp (i \pi / 6)$ is independent of $\sigma_{0}$ and it is remarkable that if $\gamma=1+i \sigma_{0} /(\omega \rho)$ is used, there exists a local minimum for the relative error around $\sigma_{0}=3500 \mathrm{~kg} /\left(\mathrm{m}^{3} \mathrm{~s}\right)$, both cases achieving the same order of relative error. If $\sigma_{0}$ is fixed and the angle of incidence is changed, both absorbing functions have the same behavior with a poor accuracy when the angle of incidence is close to $\pi / 2$, i.e, when the oblique plane wave is far from being normal.

2. Let us consider a two-dimensional closed waveguide $(0,+\infty) \times(0, a)$ with $a>0$, filled with an isotropic compressible fluid. The expression describing the motion of a fixed evanescent mode (see [60]) is given by $\phi\left(x_{1}, x_{2}\right)=\exp \left(-\beta x_{2}\right) u\left(x_{1}\right)$ where 
$\beta>0, \beta \neq \omega / c$, and $u$ is the solution of the following problem:

$$
\begin{aligned}
-\left(\rho c^{2} u^{\prime}\right)^{\prime}-\left(\omega^{2}-c^{2} \beta^{2}\right) \rho u & =0 & & \text { in }(0,+\infty), \\
u & =1 & & \text { at } x=0, \\
\rho c u^{\prime}-i \rho \sqrt{\omega^{2}-c^{2} \beta^{2}} u & =0 & & \text { at } x \rightarrow+\infty,
\end{aligned}
$$

where boundary condition (25) has been fitted to impose unit amplitude and null phase of the evanescent mode at $x=0$.

Since $\beta$ is a fixed positive constant, it can be chosen larger than $\omega / c$ and so the coefficient multiplying the mass term becomes negative. This situation can arise in other contexts as in meta-material modeling (see [31]) where the refraction index is allowed to be negative.
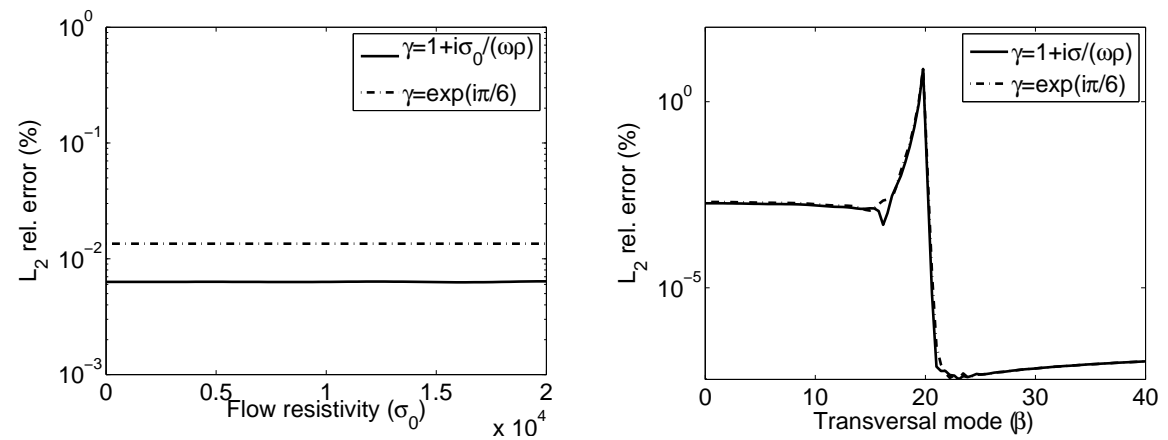

Fig. $8 \mathrm{~L}^{2}$-relative error for problem (24)-(26) varying parameter $\sigma_{0}$ with $\beta=20.2 \mathrm{~m}^{-1}$ (left) and varying the values for the transverse mode $\beta$ with fixed $\sigma_{0}=3500 \mathrm{~kg} /\left(\mathrm{m}^{3} \mathrm{~s}\right)$ (right).

The $\mathrm{L}^{2}$-relative error is shown in Figure 8 when $\sigma_{0}$ lies in the range between $0 \mathrm{~kg} /\left(\mathrm{m}^{3} \mathrm{~s}\right)$ and $2 \times 10^{4} \mathrm{~kg} /\left(\mathrm{m}^{3} \mathrm{~s}\right)$ and the transverse mode $\beta$ is fixed to $20.2 \mathrm{~m}^{-1}$. We observe that the relative error remains constant for the two different absorbing functions.

Moreover, notice that since $\omega / c=20 \mathrm{~m}^{-1}$, if the value of $\beta$ is changed then the relative error presents a singular behavior just at $\beta=20 \mathrm{~m}^{-1}$, where the problem (26)-(26) is not well-posed.

3 . The expression governing the motion of a fixed leaky mode (see [20]) in a twodimensional open waveguide $(0,+\infty) \times(0,+\infty)$ filled with an isotropic compressible fluid depends on the solution $u$ of the following problem:

$$
\begin{aligned}
-\left(\rho c^{2} u^{\prime}\right)^{\prime}-\omega^{2} \theta \rho u & =0 & & \text { in }(0,+\infty), \\
u & =1 & & \text { at } x=0, \\
\rho c u^{\prime}-i \sqrt{\omega^{2} \theta} \rho u & =0 & & \text { at } x \rightarrow+\infty,
\end{aligned}
$$

where we assume that $\theta$ is a fixed non-dimensional complex parameter such that $|\theta|=1$ and $\operatorname{Im}(\theta)<0$. In this case, the $\mathrm{L}^{2}$-relative error plots are reported in Figure 9. If we fix $\theta=\exp (-i \pi / 10)$, the solution of the PML problem roughly 

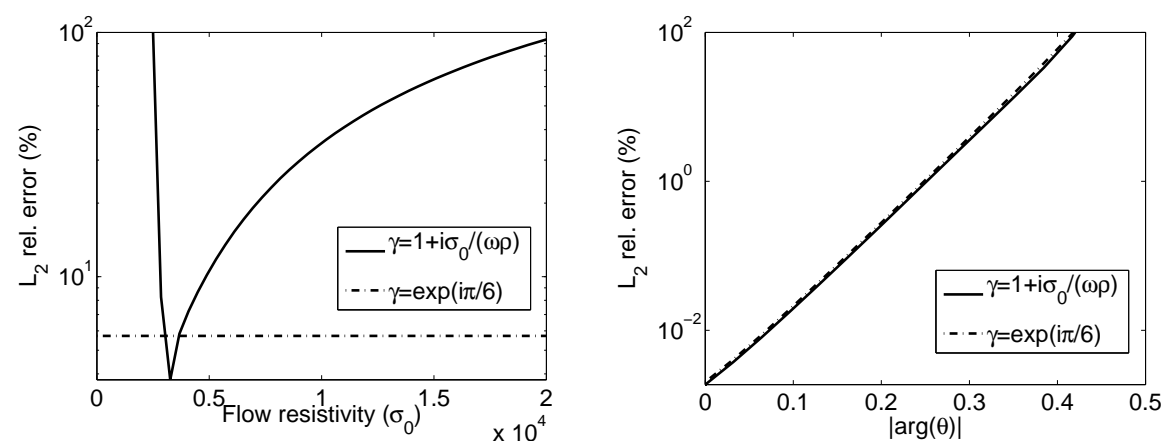

Fig. $9 \mathrm{~L}^{2}$-relative error for problem (27)-(29) varying parameter $\sigma_{0}$ with $\theta=\exp (-i \pi / 10)$ (left) and varying the phase of $\beta$ with fixed $\sigma_{0}=3500 \mathrm{~kg} /\left(\mathrm{m}^{3} \mathrm{~s}\right)$ (right).

approximate the exact solution of problem (27)-(29) for small or large values of $\sigma_{0}$, achieving only an acceptable accuracy in a small range of values of $\sigma_{0}$.

The situation becomes more dramatic if the phase of $\theta$ is close to $-\pi / 2$. In fact, it is enough to fix $\arg (\theta)>\pi / 6$ to obtain relative errors larger than $5 \%$.

These three test problems have been chosen to illustrate that, depending on the model, there are different kinds of waves that should be absorbed into the PML domain. Whereas in the first problem all waves are propagative (with a positive wavenumber), in the second one the waves are evanescent and so exponentially decreasing. In the third problem, the solution is composed of waves that are exponentially increasing with respect to the spatial variable.

Hence, even remaining valid the PML governing equation given by (20), what ensures that the characteristic impedances of the physical medium and the absorbing PML domain match, the requirements for the absorbing function $\gamma$ must be changed from one problem to another.

In fact, one of the main drawbacks of the PML technique is the lack of a general rule guaranteeing the dissipative behavior stated as the first required property in the PML conditions. In spite of this fact, for the simple one-dimensional problems that we have considered above, it is possible to write a result specifying the assumptions required to construct an accurate PML model. In what follows we describe this result.

Let us assume that $u$ is the unique solution to the problem

$$
-\left(C u^{\prime}\right)^{\prime}-A u=0 \quad \text { in }(0,+\infty)
$$

boundary conditions

at $x=0$,

$$
u^{\prime}-i \sqrt{A / C} u=0 \quad \text { at } x \rightarrow+\infty,
$$

where $A$ and $C$ are non-null constant (possibly complex and depending on the angular frequency $\omega$ ). Let us introduce the truncation of this problem by a PML domain of 
finite thickness $L$, which is described by the coupled problem

$$
\begin{aligned}
-\left(C u_{\mathrm{F}}^{\prime}\right)^{\prime}-A u_{\mathrm{F}}=0 & \text { in }(0,1), \\
-\left(\frac{C}{\gamma} u_{\mathrm{A}}^{\prime}\right)^{\prime}-A \gamma u_{\mathrm{A}}=0 & \text { in }(1,1+L), \\
\text { boundary conditions } & \text { at } x=0, \\
u_{\mathrm{F}}=u_{\mathrm{A}} & \text { at } x=1, \\
C u_{\mathrm{F}}^{\prime}=\frac{C}{\gamma} u_{\mathrm{A}}^{\prime} & \text { at } x=1 . \\
u_{\mathrm{A}}=0 & \text { at } x=1+L .
\end{aligned}
$$

In this context, we have the following lemma.

Lemma 1 Let $u$ be the unique solution to problem (30)-(32) and $\left(u_{\mathrm{F}}, u_{\mathrm{A}}\right)$ the unique solution to PML problem (33)-(38). If $\operatorname{Im}\left(\sqrt{A / C} \int_{1}^{L+1} \gamma(s) \mathrm{d} s\right)$ is a positive nondecreasing function with respect to $L$, then fixed $L_{0}>0$, there exists a positive constant $M$ independent of $L$ and $\gamma$ such that

$$
\int_{0}^{L}\left|u(x)-u_{\mathrm{F}}(x)\right|^{2} \mathrm{~d} x \leq M e^{-4 \operatorname{Im}\left(\sqrt{\frac{A}{C}} \int_{1}^{L+1} \gamma(s) \mathrm{d} s\right)},
$$

for all $L>L_{0}$

The sketch of the proof is as follows. The solution of problem (30)-(32) is $u(x)=$ $A e^{i k_{\mathrm{F}}(x-1)}+B e^{-i k_{\mathrm{F}}(x-1)}$, with $k_{\mathrm{F}}=\sqrt{A / C}$. Since any boundary condition at $x=0$ can be written in terms of the amplitudes $A$ and $B$ as $r A+s B=t$, where $t$ is an arbitrary constant and $r \neq 0$ and $s$ are complex-valued constants depending on $k_{\mathrm{F}}$. Hence, the amplitude of the solutions is fixed by the boundary conditions to $A=$ $(t / r) e^{i k_{\mathrm{F}}}$ and $B=0$.

Taking into account the differential equations (33) and (34) and the boundary conditions (35) and (36), the solution of the PML problem is given by $u_{\mathrm{F}}(x)=$ $\tilde{A} e^{i k_{\mathrm{F}}(x-1)}+\tilde{B} e^{-i k_{\mathrm{F}}(x-1)}$ and $u_{\mathrm{A}}(x)=\tilde{A} e^{i k_{\mathrm{F}} \int_{1}^{x} \gamma(s) d s}+\tilde{B} e^{-i k_{\mathrm{F}} \int_{1}^{x} \gamma(s) d s}$. If we impose the boundary conditions at $x=0$ and $x=L+1$, we obtain

$$
\tilde{A}=\frac{\frac{t}{r} e^{i k_{\mathrm{F}}}}{1-\frac{s}{r} e^{2 i k_{\mathrm{F}}} e^{2 i k_{\mathrm{F}} \int_{1}^{L+1}} \gamma(s) d s}, \quad \tilde{B}=-\frac{\frac{t}{r} e^{i k_{\mathrm{F}}} e^{2 i k_{\mathrm{F}} \int_{1}^{L+1} \gamma(s) d s}}{1-\frac{s}{r} e^{2 i k_{\mathrm{F}}} e^{2 i k_{\mathrm{F}} \int_{1}^{L+1} \gamma(s) d s}},
$$

where the denominators are not null since we have assumed that the solution $\left(u_{\mathrm{F}}, u_{\mathrm{A}}\right)$ is unique.

Since $\left|e^{2 i k_{\mathrm{F}} \int_{1}^{L+1}} \gamma(s) d s\right|=e^{-2 \operatorname{Im}\left(k_{\mathrm{F}} \int_{1}^{L+1} \gamma(s) d s\right)}$, the modulus of the denominators in (39) are increasing functions which tends to one when $L \rightarrow \infty$, and so they remain lower bounded by a constant $M_{1}$ for all $L>L_{0}$. Moreover, from (39) it is easy to obtain $|A-\tilde{A}| \leq t s /\left(r^{2} M_{1}\right) e^{-2 \operatorname{Im}\left(k_{\mathrm{F}} \int_{1}^{L+1} \gamma(s) d s\right)}$ and $|\tilde{B}| \leq t /\left(r M_{1}\right) e^{-2 \operatorname{Im}\left(k_{\mathrm{F}} \int_{1}^{L+1} \gamma(s) d s\right)}$. Finally, taking into account that there exists a positive constant $M_{2}$ such that

$$
\int_{0}^{L}\left|u(x)-u_{\mathrm{F}}(x)\right|^{2} \mathrm{~d} x \leq M_{2}\left(|A-\tilde{A}|^{2}+|\tilde{B}|^{2}\right),
$$

the estimate of the lemma is concluded. 
Now, we are able to reinterpret the results shown in the three test problems above thanks to Lemma 1. In the first problem, since the wavenumber $\sqrt{C / A}=\omega \cos (\theta) / c$ is always positive and tends to zero as $\theta \rightarrow \pi / 2$, the relative error is allowed to increase when $\theta \rightarrow \pi / 2$. In the second problem, if $\beta>\omega / c$ then the wavenumber $\sqrt{C / A}$ is pure imaginary and so the exponential decay coefficient of the truncation error only depends on the real part of $\gamma$. Hence, this fact explains why the relative error is independent of $\sigma_{0}$ in the left plot of Figure 8.

In the case of the third test problem, since $\sqrt{C / A}$ has a negative imaginary part, a straightforward computation shows that only if $-\arg (\theta)<2 \arg (\gamma)$, the relative error is exponentially decreasing with respect to the thickness of the PML. Otherwise, the estimate given in Lemma 1 blows up exponentially and the approximation $u_{\mathrm{F}}$ becomes totally spurious what happens in this problem for $\arg (\theta)<-\pi / 6$.

Despite Lemma 1, some tasks in this first one-dimensional analysis remain open. From the figures shown in Section 2 and 3, it is concluded that the relative errors are quite sensitive to the profile chosen for the absorbing function $\gamma$ (and, in particular, for $\sigma)$. For instance, it is remarkable that as naive as it looks, the choice $\gamma=\exp (i \pi / 6)$ keeps a robust behavior in most of the problems presented in this paper and it also leads to reasonable accurate results.

However, just the choice of the absorbing function in the PML model is one of the most relevant topics for this numerical technique in time-harmonic problems. In fact, our main contribution focuses on this aspect and will be described in Section 6, in the context of the two-dimensional Helmholtz equation.

\section{PML and the analytic dilatation technique}

In the previous two sections, the PML governing equation has been derived for onedimensional problems via a modification of the Darcy model and its damping behavior has been ensured by Lemma 1. However, to extend the PML technique to two dimensional problems involving other models different from the acoustic one, such simple mechanical derivation is not useful.

With this extensional purpose, in this section we derive the PML governing equation by using a complex stretching of the spatial coordinates. We recover the same PML equation as in the one-dimensional case and, furthermore, we extend the PML technique to models that involve general second order partial differential equations.

\subsection{One-dimensional dilatation analytic technique}

In the context of the PML literature, Chew and Weedon [25] were the first authors to think up the construction of the PML governing equation by using a complex stretching of the spatial variables. An analogous strategy, called complex scaling or analytic dilatation technique, had been already developed some decades ago for the Schrodinger equation. In fact, nowadays this technique is still used to simulate numerically dispersion in quantum systems (see [45] and references therein).

We sketch briefly the procedure for writing the dilatation analytic technique in the one-dimensional problem (30)-(32) and, thereby, for introducing the PML governing equation through a complex stretching of coordinates. 
Let us define the complex spatial change of variable $S(x)=1+\int_{1}^{x} \gamma(s) \mathrm{d} s$ for $x \in(1,1+L)$ and let us also assume that the coefficients $A$ and $C$ of problem (30)-(32) are functions that depends analytically on the spatial variable $x$ and, additionally, $1 / C$ is well defined and also analytic. Under these conditions, the solution $u$ is analytic so it can be evaluated along the complex path $S(0,+\infty)$.

In fact, since $u$ is analytic and satisfies $(30)$ in $(1,1+L)$, then it also satisfies this ordinary differential equation in $S(1,1+L)$, namely,

$$
-\frac{d}{d \tilde{x}}\left(C \frac{d u}{d \tilde{x}}\right)-A u=0 \quad \text { in } S(1,1+L)
$$

where the spatial derivatives with respect to the variable $x$ have been replaced by the derivatives with respect to $\tilde{x}=S(x)$ and coefficients $A$ and $C$ are properly evaluated on $S(1,1+L)$.

Since $\tilde{x}$ is complex, the spatial derivatives with respect to $\tilde{x}$ must be understood in the usual Cauchy-Riemann sense (see [70]), i.e., $d u / d \tilde{x}=d \operatorname{Re} u / d s-i d \operatorname{Im} u / d t$ where $s=\operatorname{Re} \tilde{x}$ and $t=\operatorname{Im} \tilde{x}$.

To rewrite the previous equation stated in the original real interval $(1,1+L)$, it is enough to apply the chain's rule. If coefficients $A$ and $C$ are constant, $A \circ S=A$ and $C \circ S=C$. Moreover, if we assume additionally that $\gamma$ does not vanish in $(1,1+L)$ and since $S^{\prime}(x)=\gamma(x)$, we recover (34) in the absorbing domain $(1,1+L)$ which has been claimed as the one-dimensional general PML governing equation in the section above.

4.2 PML governing equation for second order PDEs

Now, we follow the same one-dimensional idea based on the complex coordinate stretching in a more general framework. As we have checked, the description of the PML governing equation is related to an analytic dilatation of the original equation in onedimensional problems. In what follows we detail the same analytic dilatation procedure to write the PML equation in two dimensions.

We restrict ourselves to the two-dimensional case and to models which are described by second order partial differential equations with only mass and stiffness terms. However, analogous constructions could be done for the three-dimensional case and for higher order differential equations.

To write the partial differential equation of this general model, tensors and vector fields are described by using the Cartesian reference system with canonical basis $\left\{\mathbf{e}_{1}, \mathbf{e}_{2}\right\}$. Thus, if the contrary is not explicitly specified, vector and tensor coordinates are given in the Cartesian reference system.

We also assume the Einstein convention that the repeated subscript adds. Moreover, given a $\mathbb{C}^{2}$-vector field $\mathbf{v}$, the partial derivative of the component $i$ with respect to the $j$-th variable is denoted by $v_{i, j}$, where $1 \leq i \leq 2$ and $j=1,2$.

Let us assume that the second and fourth order linear tensors $A$ and $C$ have coefficients (possibly complex and depending on the angular frequency $\omega$ ), which are analytic functions with respect to $\mathbf{x}$.

In this general framework, let us also introduce a compact domain $O$ with smooth boundary $\partial O$, which plays the role of a scatter. The general second order scattering problem is stated in the exterior of the bounded domain $O$ and described by the following set of equations: 
Given the angular frequency $\omega>0$ and $a \mathbb{C}^{2}$-vector source $\mathbf{f}$ with compact support, find the $\mathbb{C}^{2}$-vector field $\mathbf{u}$ such that

$$
\begin{aligned}
-\left(C_{i j k l} u_{k, l}\right)_{, j}-A_{i j} u_{j}=f_{i} & \text { in } \mathbb{R}^{2} \backslash \bar{O}, i=1,2, \\
C_{i j k l} u_{k, l} n_{j}=0 & \text { on } \partial O, i=1,2, \\
\text { radiation conditions } & \text { at }|\mathbf{x}| \rightarrow \infty,
\end{aligned}
$$

where $\mathbf{n}$ is the unit normal vector on $\partial O$ outward to $O$.

Obviously, for every particular problem, different radiation conditions should be specified depending on $A$ and $C$ to guarantee the right decay behavior of the solution at infinity and to get the uniqueness of solution. In what follows we assume that (40)(42) have a unique solution.

Now we are in a position to describe the PML technique corresponding to this second order problem. Since the domain of this problem, $\Omega=\mathbb{R}^{2} \backslash \bar{O}$, is unbounded and our attention is restricted to compute the solution only in a bounded region of interest, namely, the physical domain $\Omega_{\mathrm{F}}$, we place the PML absorbing medium $\Omega_{\mathrm{A}}$ surrounding the region of interest (see Figure 10). At this stage, no considerations will be done about the boundedness of the PML domain, which is not relevant for the derivation of the PML governing equation.

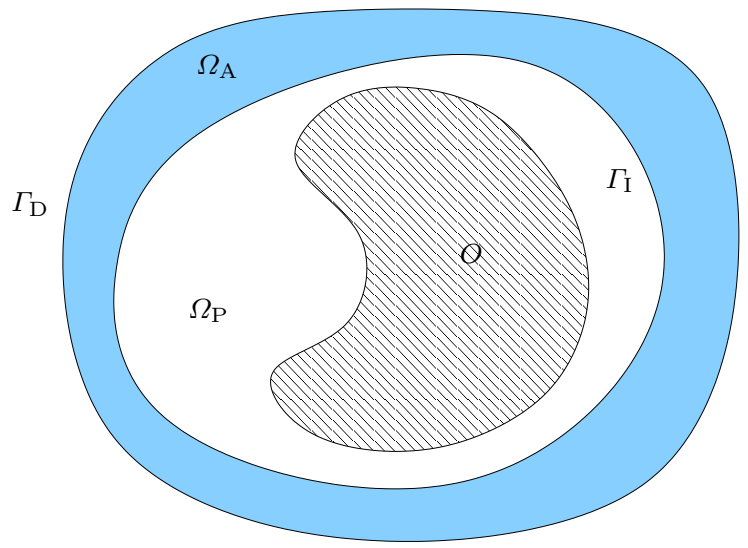

Fig. 10 General convex domain $\Omega_{\mathrm{B}}$ for the PML problem.

It is also useful to introduce the notation of $\Gamma_{\mathrm{D}}$ and $\Gamma_{\mathrm{I}}$ for the exterior boundary of the PML domain and the interface between $\Omega_{\mathrm{F}}$ and $\Omega_{\mathrm{A}}$, respectively. Moreover, the union of physical and PML domain will be denoted by $\Omega_{\mathrm{B}} \subset \Omega$.

Associated to the construction of the PML equation, we are going to define a complex change of variable $\mathbf{S}: \mathbf{x} \in \Omega_{\mathrm{B}} \subset \mathbb{R}^{2} \rightarrow \mathbf{S}(\mathbf{x})=\tilde{\mathbf{x}} \in \mathbf{S}\left(\Omega_{\mathrm{B}}\right) \subset \mathbb{C}^{2}$ and then to introduce the PML problem as a 'complexified' version of problem (40)-(42).

Since we want to keep the original solution in the physical region of interest $\Omega_{\mathrm{F}} \subset$ $\Omega_{\mathrm{B}}$ which contains the support of the source term defined by $\mathbf{f}$, we assume that $\mathbf{S}(\mathbf{x})=$ $\mathbf{x}$ in $\Omega_{\mathrm{F}}$, whereas it is complex-valued outside $\Omega_{\mathrm{F}}$.

First, we introduce the weak problem associated with (40)-(42). If we multiply (40) by the conjugate of a complex vector field $\mathbf{v}$ of compact support containing $\Omega_{\mathrm{F}}$, we 
obtain

$$
\int_{\Omega_{\mathrm{B}}}\left(C_{i j k l} u_{k, l} \bar{v}_{i, j}-A_{i j} u_{j} \bar{v}_{i}\right) \mathrm{d} V=\int_{\Omega_{\mathrm{B}}} f_{i} \bar{v}_{i} \mathrm{~d} V .
$$

In what follows, we assume that the solution $\mathbf{u}$ and the test functions and their partial derivatives admit an extension in the closure of $\mathbf{S}\left(\Omega_{\mathrm{A}}\right)$. Hence, as it has been done for the one-dimensional problem, we are able to evaluate $\mathbf{u}$ and $\mathbf{v}$ at points $\tilde{\mathbf{x}} \in \mathbf{S}\left(\Omega_{\mathrm{B}}\right)$, and so the analytic continuation of $\mathbf{u}$ makes sense in $\mathbf{S}\left(\Omega_{\mathrm{B}}\right)$.

Moreover, we can replace formally the spatial derivatives with respect to coordinates $\mathbf{x}$ by derivatives with respect to $\tilde{\mathbf{x}}$. Analogous to the notation introduced above, $v_{i, \tilde{j}}$ denotes the partial derivative of the $i$-th component with respect to the $j$-th coordinate of $\tilde{\mathbf{x}}$. Consequently, we have

$$
\int_{\mathbf{S}\left(\Omega_{\mathrm{B}}\right)}\left(C_{i j k l} u_{k, \tilde{l}} \bar{v}_{i, \tilde{j}}-A_{i j} u_{j} \bar{v}_{i}\right) \mathrm{d} \tilde{V}=\int_{\Omega_{\mathrm{B}}} f_{i} \bar{v}_{i} \mathrm{~d} V
$$

Let us remark that since $\mathbf{S}(\mathbf{x})=\mathbf{x}$, then $\mathbf{S}\left(\Omega_{\mathrm{F}}\right)=\Omega_{\mathrm{F}}$ and so the source term in the right-hand side remains equal in the analytic continuation of the weak problem.

Then, we rewrite the previous weak problem coming back to the real-valued domain $\Omega_{\mathrm{B}}$ applying the chain's rule for $\mathbf{S}$. More precisely, let us assume that $\mathbf{S}$ is smooth and inversible in $\Omega_{\mathrm{B}}$, we have

$$
\begin{aligned}
\int_{\Omega_{\mathrm{B}}}\left((C \circ \mathbf{S})_{i j k l}(u \circ \mathbf{S})_{k, n}\left(H^{-1}\right)_{n l}(\overline{\mathbf{v}} \circ \mathbf{S})_{i, m}\left(H^{-1}\right)_{m j}\right. & \\
& \left.-(A \circ \mathbf{S})_{i j}(\mathbf{u} \circ \mathbf{S})_{j}(\overline{\mathbf{v}} \circ \mathbf{S})_{i}\right) \operatorname{det} H \mathrm{~d} V=\int_{\Omega_{\mathrm{B}}} f_{i} \bar{v}_{i}, \mathrm{~d} V,
\end{aligned}
$$

where $H$ is the Jacobian matrix of $\mathbf{S}$.

Now, if we introduce the notation $\tilde{\mathbf{u}}=\mathbf{u} \circ \mathbf{S}$ and $\tilde{\mathbf{v}}=\mathbf{v} \circ \mathbf{S}$, we have

$$
\begin{aligned}
\int_{\Omega_{\mathrm{B}}}\left((C \circ \mathbf{S})_{i j k l}\left(H^{-1}\right)_{n l}\left(H^{-1}\right)_{m j} \tilde{u}_{k, n} \overline{\tilde{v}}_{i, m}\right. & \\
& \left.-(A \circ \mathbf{S})_{i j} \tilde{u}_{j} \overline{\tilde{v}}_{i}\right) \operatorname{det} H \mathrm{~d} V=\int_{\Omega_{\mathrm{B}}} f_{i} \overline{\tilde{v}}_{i}, \mathrm{~d} V .
\end{aligned}
$$

Finally, integrating by parts, we obtain the partial differential equation governing the propagation in the PML problem, written in divergence form as

$$
-\left(\tilde{C}_{i j k l} \tilde{u}_{k, l}\right)_{, j}-\tilde{A}_{i j} \tilde{u}_{j}=f_{i} \quad \text { in } \Omega_{\mathrm{B}}, i=1,2,
$$

where $\tilde{A}_{i j}=(\operatorname{det} H)(A \circ \mathbf{S})_{i j}$ and $\tilde{C}_{i j k l}=(\operatorname{det} H)(C \circ \mathbf{S})_{i m k n}\left(H^{-1}\right)_{j m}\left(H^{-1}\right)_{l n}$.

Although we have required that $\mathbf{S}$ is smooth, we will see in Section 5 that, in general, the coefficients of tensor $\tilde{C}$ can be discontinuous. In this case, we must understand that $\tilde{\mathbf{u}}$ satisfies (44) in a weak sense, i.e., if and only if $\tilde{\mathbf{u}}$ satisfies (43) for any $\tilde{\mathbf{v}}$ with compact support in $\Omega_{\mathrm{B}}$.

Equivalently, we could also introduce a coupled formulation, as we have already done for (30)-(32) in the one-dimensional case, and specify explicitly that $\tilde{u}_{i}$ and $\tilde{C}_{i j k l} \tilde{u}_{k, l} n_{j}$ are continuous on $\Gamma_{\mathrm{I}}$.

The PML governing equation given by (44) will be considered as the general framework throughout the rest of the paper. Let us remark that, since $\mathbf{S}(\mathbf{x})=\mathbf{x}$ in $\Omega_{\mathrm{F}}$, the source term in (44) remains unchanged with respect to (40). 
A direct inspection of the construction described above shows that, since $\mathbf{u}$ is an analytic solution satisfying the partial differential equation (40) and the boundary condition (41), then $\tilde{\mathbf{u}}$, i.e, $\mathbf{u} \circ \mathbf{S}$, satisfies the same boundary condition (41) and the partial differential equation (44).

Let us recall that until now we have only derived the governing equation of the PML but not the complete PML problem which is going to replace (40)-(42). Moreover, the derivation of (44) has been done independently of possible requirements either on the signs of the real and imaginary part of the complex stretching of variables $\mathbf{S}$ or on the geometry of the absorbing domain $\Omega_{\mathrm{A}}$.

Notice also that, at this point, it does not exist any restriction to be assumed about the complex change of variable $\mathbf{S}$ which can be eventually dependent on $\omega$. In fact, this dependency becomes useful to obtain a uniform exponential decay of the truncation error with respect to the angular frequency (see Section 5 for details).

Finally, let us remark that the assumption of analyticity for coefficients $A$ and $C$ has been done to ensure that $A_{i j} \circ \mathbf{S}$ and $C_{i j k l} \circ \mathbf{S}$ are well defined. However, this assumption can be made milder by only requiring that $A_{i j}$ and $C_{i j k l}$ are analytic in the two-dimensional complex manifold $\mathbf{S}\left(\Omega_{\mathrm{B}}\right)$.

\subsection{Cartesian and radial PML equation}

Now, we are in a position to recover the PML equations in the particular cases where the change of variable uses Cartesian and radial coordinates. In the literature, there are a wide variety of PML models using different local coordinate systems as cylindrical [65] or spherical coordinates [76],[77] in the three-dimensional case or, with more generality, a combination of those [80], a conformal transformation of coordinates [58], or even using polygonal convex coordinates locally [83].

For the sake of brevity, we only describe the most usual complex coordinates stretching in the context of the general second order elliptic operators.

Let us start revising the standard Cartesian PML equation governing the motion in a corner domain $\Omega_{\mathrm{A}}=\left\{\mathbf{x} \in \mathbb{R}^{2}: x_{1}>L_{1}, x_{2}>L_{2}\right\}$. For this geometry, we introduce the following complex change of variable:

$$
\mathbf{S}(\mathbf{x})= \begin{cases}\left(L_{1}+\int_{L_{1}}^{x_{1}} \gamma_{1}(s) \mathrm{d} s, L_{2}+\int_{L_{2}}^{x_{2}} \gamma_{2}(s) \mathrm{d} s\right) & \text { if } \mathbf{x} \in \Omega_{\mathrm{A}} \\ \mathbf{x} & \text { otherwise }\end{cases}
$$

where $\gamma_{1}, \gamma_{2}$ are the so-called absorbing functions in the $x_{1}$ and $x_{2}$-directions, respectively. These functions are a priori complex-valued and no restriction are imposed to obtain the "perfectly matched" behavior between the physical domain and the PML region. Taking into account this complex coordinate stretching, the Jacobian matrix $H$ is diagonal,

$$
H_{i j}= \begin{cases}\gamma_{i} \delta_{i j} & \text { in } \Omega_{\mathrm{A}} \\ \delta_{i j} & \text { otherwise. }\end{cases}
$$


and so we have

$$
\begin{aligned}
\tilde{A}_{i j} & = \begin{cases}\gamma_{1} \gamma_{2} A_{i j} & \text { in } \Omega_{\mathrm{A}}, \\
A_{i j} & \text { otherwise, }\end{cases} \\
\tilde{C}_{i j k l} & = \begin{cases}\frac{\gamma_{1} \gamma_{2}}{\gamma_{j} \gamma_{l}} C_{i j k l}, & \text { in } \Omega_{\mathrm{A}}, \\
C_{i j k l} & \text { otherwise },\end{cases}
\end{aligned}
$$

where no summation arise on indices $j$ and $l$.

In the case of the radial PML, the deduction is also straightforward from the complex change of variable. If we introduce the radial and angular coordinates $r=|\mathbf{x}|$ and $\theta=\arg (\mathbf{x})$, and assume that the PML domain is placed surrounding the ball of radius $R$, i.e., $\Omega_{\mathrm{A}}=\left\{\mathbf{x} \in \mathbb{R}^{2}:|\mathbf{x}|=r>R\right\}$, we have

$$
\mathbf{S}(\mathbf{x})= \begin{cases}\left(R+\int_{R}^{r} \gamma_{r}(s) \mathrm{d} s\right)(\cos \theta, \sin \theta), & \text { if } \mathbf{x} \in \Omega_{\mathrm{A}} \\ \mathbf{x} & \text { otherwise }\end{cases}
$$

where $\gamma_{r}$, is again the so-called absorbing function in the radial direction. Analogous to the Cartesian case, $\gamma_{r}$ is a complex-valued function without any special requirements. After some algebraic manipulations, this complex change of variable leads to

$$
H=\left(\begin{array}{cc}
\cos \theta & \sin \theta \\
-\sin \theta & \cos \theta
\end{array}\right)^{t}\left(\begin{array}{cc}
\gamma_{r} & 0 \\
0 & \tilde{\gamma}
\end{array}\right)\left(\begin{array}{cc}
\cos \theta & \sin \theta \\
-\sin \theta & \cos \theta
\end{array}\right),
$$

where $\tilde{\gamma}=\frac{1}{r}\left(R+\int_{R}^{r} \gamma_{r}(s) \mathrm{d} s\right)$.

In order to obtain simple expressions of coefficients $\tilde{A}_{i j}$ and $\tilde{C}_{i j k l}$ for the PML model, it is necessary to write tensors and vector fields in terms of the polar canonical vector basis $\left\{\mathbf{e}_{r}, \mathbf{e}_{\theta}\right\}$. In this basis, the Jacobian matrix $H$ becomes diagonal and its expression is again given by (46) where $\gamma_{1}$ and $\gamma_{2}$ must be replaced by $\gamma_{r}$ and $\tilde{\gamma}$, respectively.

If, for the sake of simplicity, we abuse of notation and use the same variables for the radial coordinates of the tensor and vector fields, the PML governing equation involves the same coordinate tensors in vector basis $\left\{\mathbf{e}_{r}, \mathbf{e}_{\theta}\right\}$ given by (47)-(48), where we recall that we should read $\gamma_{1}=\gamma_{r}$ and $\gamma_{2}=\tilde{\gamma}$ for the radial PML.

Let us remark that in the Cartesian and radial cases, since the Jacobian matrix $H$ is diagonal respect to the canonical bases $\left\{\mathbf{e}_{1}, \mathbf{e}_{2}\right\}$ and $\left\{\mathbf{e}_{r}, \mathbf{e}_{\theta}\right\}$, respectively, then no summations arise in the formulas for the coefficients of $\tilde{A}$ and $\tilde{C}$. This issue has special relevance from a implementation point of view, since the inclusion of the PML technique only requires to multiply the implemented PDE coefficients by some complex-valued factors.

However, in a hypothetical general case where each component of the complex coordinate stretching $\mathbf{S}$ depends on all spatial coordinates, it is not possible to choose a local orthogonal system of reference for which the Jacobian matrix $H$ becomes diagonal.

\subsection{Some examples}

Now, we apply the formal construction of the PML governing equation to obtain wellknown Cartesian PML models in simple cases such as the pressure and displacement formulations for the motion of an isotropic acoustic fluid from the rest, and the PML 
model for isotropic linear elastic materials. Analogous considerations could be done for more sophisticated models such as the linearized Euler equation or those modeling viscoelastic or poroelastic materials.

1. The time-harmonic motion of an isotropic compressible fluid at rest under the action of a volumetric source term $f$ can be described in terms of the scalar pressure field $p$ by

$$
-c^{2} \operatorname{div} \operatorname{grad} p-\omega^{2} p=f,
$$

being $\omega$ the angular frequency and $c$ the sound speed. A direct inspection of this equation leads to $A_{11}=-\omega^{2}$ and $C_{1 j 1 l}=c^{2} \delta_{j l}$ for $j, l=1,2$ in the physical domain. If we apply formulas (47)-(48) for the Cartesian PML technique then we have $\tilde{A}_{11}=-\omega^{2} \gamma_{1} \gamma_{2}, \tilde{C}_{1111}=-c^{2} \gamma_{2} / \gamma_{1}$ and $\tilde{C}_{1212}=-c^{2} \gamma_{1} / \gamma_{2}$ in the absorbing medium $\Omega_{\mathrm{A}}$. This set of expressions yields the PML governing equation:

$$
-c^{2} \operatorname{div}\left(\left(\begin{array}{cc}
\gamma_{2} / \gamma_{1} & 0 \\
0 & \gamma_{1} / \gamma_{2}
\end{array}\right) \operatorname{grad} \tilde{p}\right)-\omega^{2} \gamma_{1} \gamma_{2} \tilde{p}=0,
$$

which is the well-known Cartesian PML equation for the Helmholtz equation.

2. The description of time-harmonic motion in a isotropic compressible fluid at rest in terms of the displacement vector field is given by the following equation:

$$
-\operatorname{grad}\left(\rho c^{2} \operatorname{div} \mathbf{u}\right)-\omega^{2} \rho \mathbf{u}=\mathbf{f}
$$

where $\rho$ is the mass density and, again, $\omega$ and $c$ are the angular frequency and the sound speed, respectively. If we rewrite the previous equation using the general second order form (40) and use (47), (48) we obtain the following PML coefficients: $\tilde{A}_{11}=\tilde{A}_{22}=-\omega^{2} \rho \gamma_{1} \gamma_{2}, \tilde{C}_{1111}=-\rho c^{2} \gamma_{2} / \gamma_{1}, \tilde{C}_{2222}=-\rho c^{2} \gamma_{1} / \gamma_{2}$, and $\tilde{C}_{1122}=$ $\tilde{C}_{2211}=-\rho c^{2}$. Hence, the resulting PML equation is given by

$$
-\operatorname{grad}\left(\left(\begin{array}{cc}
\rho c^{2} \gamma_{2} / \gamma_{1} & \rho c^{2} \\
\rho c^{2} & \rho c^{2} \gamma_{1} / \gamma_{2}
\end{array}\right)\left(\begin{array}{c}
\tilde{u}_{1,1} \\
\tilde{u}_{2,2}
\end{array}\right)\right)-\omega^{2} \rho \gamma_{1} \gamma_{2} \tilde{\mathbf{u}}=\mathbf{0} .
$$

Analogous to the relation between (50) and (52), where $p$ can be written as $p=$ $-\rho c^{2} \operatorname{div} \mathbf{u}$, it is remarkable that it is possible to recover the pressure field $\tilde{p}$ satisfying (51) from the displacement field $\tilde{\mathbf{u}}$ in (53) by

$$
\tilde{p}=-\rho c^{2}\left(\frac{1}{\gamma_{1}} \tilde{u}_{1,1}+\frac{1}{\gamma_{2}} \tilde{u}_{2,2}\right) .
$$

3. Under the assumption of isotropy, the time-harmonic motion of an elastic material modeled by the linear Hooke's constitutive law is described, in terms of the displacement field $\mathbf{u}$, as

$$
-\operatorname{div} \Sigma(\mathbf{u})-\omega^{2} \rho \mathbf{u}=\mathbf{f},
$$

where $\Sigma(\mathbf{u})_{i j}=\lambda \delta_{i j} E(\mathbf{u})_{k k}+2 \mu E(\mathbf{u})_{i j}$, being $E(\mathbf{u})_{i j}=(1 / 2)\left(u_{i, j}+u_{j, i}\right)$ and $\lambda$ and $\mu$ the so called Lame's coefficients. Again, a simple computation shows that the previous equation is a particular case of (40) with tensors $A_{i j}=-\omega^{2} \delta_{i j}$ and $C_{i j k l}=\lambda \delta_{i j} \delta_{l k}+\mu\left(\delta_{i k} \delta_{j l}+\delta_{i l} \delta_{j k}\right)$. In this case, the index symmetries of tensor $C$, namely, $C_{i j k l}=C_{k l i j}=C_{j i k l}=C_{i j l k}$ allows us to reduce the description of all the tensor coefficients to nine of them. However, we use a redundant description which 
involves sixteen. If the following index mapping is used: $\{11\} \leftrightarrow\{1\},\{22\} \leftrightarrow\{2\}$, $\{12\} \leftrightarrow\{3\},\{21\} \leftrightarrow\{4\}$, then tensor $C$ is described by the $4 \times 4$ matrix

$$
\left(\begin{array}{cccc}
2 \mu+\lambda & \lambda & 0 & 0 \\
\lambda & 2 \mu+\lambda & 0 & 0 \\
0 & 0 & \mu & \mu \\
0 & 0 & \mu & \mu
\end{array}\right)
$$

A direct application of formulas (47)-(48) shows that $\tilde{A}_{11}=\tilde{A}_{22}=-\omega^{2} \rho \gamma_{1} \gamma_{2}$ and hence tensor $\tilde{C}$ is described by the matrix

$$
\left(\begin{array}{cccc}
\frac{\gamma_{2}}{\gamma_{1}}(2 \mu+\lambda) & \lambda & 0 & 0 \\
\lambda & \frac{\gamma_{1}}{\gamma_{2}}(2 \mu+\lambda) & 0 & 0 \\
0 & 0 & \frac{\gamma_{2}}{\gamma_{1}} \mu & \mu \\
0 & 0 & \mu & \frac{\gamma_{1}}{\gamma_{2}} \mu
\end{array}\right)
$$

Indeed, it is checked that the index symmetry $\{12\} \leftrightarrow\{21\}$, which is verified by tensor $C$, is lost for the PML tensor $\tilde{C}$. From a physical point of view, this nonsymmetry can be associated to an anisotropic material where the stress tensor depends on the full gradient of $\mathbf{u}$ (see [43] for details).

\section{Theoretical results using bounded absorbing functions}

In the previous section we have described the construction of the PML governing equation for models involving second order partial differential equations. Now we complete the description of the PML problem assuming that the computational domain $\Omega_{\mathrm{B}}$ is bounded and imposing homogeneous Dirichlet boundary conditions on the exterior boundary $\Gamma_{\mathrm{D}}$.

Hence, problem (40)-(42), originally stated in an unbounded domain, is going to be replaced by the following PML problem:

Given the angular frequency $\omega>0$ and $a \mathbb{C}^{2}$-vector source $\mathbf{f}$ with compact support, find the $\mathbb{C}^{2}$-vector field $\tilde{\mathbf{u}}$ such that

$$
\begin{aligned}
-\left(\tilde{C}_{i j k l} \tilde{u}_{k, l}\right)_{, j}-\tilde{A}_{i j} \tilde{u}_{j}=f_{i} & \text { in } \Omega_{\mathrm{B}}, i=1,2, \\
\tilde{C}_{i j k l} \tilde{u}_{k, l} n_{j}=0 & \text { on } \partial O, i=1,2, \\
\tilde{u}_{i}=0 & \text { on } \Gamma_{\mathrm{D}} .
\end{aligned}
$$

Obviously, it would be desirable to have a result analogous to Lemma 1 where the $\mathrm{L}^{2}$-error between $\tilde{\mathbf{u}}$ and the solution $\mathbf{u}$ of problem (40)-(42) were estimated theoretically. Unfortunately, the mathematical analysis of PML problem (57)-(59) is far from being as simple as in the one-dimensional case. In fact, before studying the estimates between $\mathbf{u}$ and $\tilde{\mathbf{u}}$, we should ensure that there exists a unique solution for the PML problem (57)-(59). 
5.1 Existence and uniqueness of solution

In this section, we restrict ourselves to those cases where the absorbing functions in the PML domain $\Omega_{\mathrm{A}}$ are bounded. Later, in Section 6 , we will describe a particular case where unbounded absorbing functions are used.

First, we must precise the standard functional framework to study the PML problem (57)-(59). We recall the definition of some function spaces which will be used throughout this section. Let $\mathrm{L}_{2}\left(\Omega_{\mathrm{B}}\right)$ be the space of square integrable complex valued functions defined in $\Omega_{\mathrm{B}}$,

$$
\mathrm{L}_{2}\left(\Omega_{\mathrm{B}}\right)=\left\{f: \Omega_{\mathrm{B}} \rightarrow \mathbb{C}: \int_{\Omega_{\mathrm{B}}}|f|^{2}<\infty\right\} .
$$

We denote by $\mathrm{H}^{1}\left(\Omega_{\mathrm{B}}\right)$ the Sobolev space of functions belonging to $\mathrm{L}_{2}\left(\Omega_{\mathrm{B}}\right)$ together with all their first order derivatives:

$$
\mathrm{H}^{1}\left(\Omega_{\mathrm{B}}\right)=\left\{f \in \mathrm{L}_{2}\left(\Omega_{\mathrm{B}}\right): \operatorname{grad} f \in \mathrm{L}_{2}\left(\Omega_{\mathrm{B}}\right)^{2}\right\} .
$$

Finally, the subspace of functions in $\mathrm{H}^{1}\left(\Omega_{\mathrm{B}}\right)$ vanishing on $\Gamma_{\mathrm{D}}$ is denoted by $\mathrm{H}_{\Gamma_{\mathrm{D}}}^{1}\left(\Omega_{\mathrm{B}}\right)$

Moreover, we have to state the hypothesis for the complex stretching of the spatial variables, $\mathbf{S}$ and its Jacobian matrix $H$. We assume that $\mathbf{S}$ depends analytically on the angular frequency and that the following inequalities hold,

$$
\max _{\mathbf{x} \in \Omega_{\mathrm{B}}}\left|S_{i}(\mathbf{x})\right|<\infty, \quad \max _{\mathbf{x} \in \Omega_{\mathrm{B}}}\left|H_{i j}(\mathbf{x})\right|<\infty, \quad \max _{\mathbf{x} \in \Omega_{\mathrm{B}}}\left|\left(H^{-1}\right)_{i j}(\mathbf{x})\right|<\infty .
$$

Additionally, we also impose that $H$ can be explicitly written as

$$
H=U^{t} \Lambda U
$$

where $U$ is a real unitary matrix and $\Lambda$ is a non-singular complex diagonal matrix, which eventually can have variable coefficients depending on the spatial variables.

This last assumption is related to restrict the complex stretching $\mathbf{S}$ to those mappings whose components only depend on one of the spatial coordinates in a local orthogonal system of reference, as in the Cartesian and polar cases presented in Subsection 4.3, or, more generally, in systems of reference derived from a convex geometry (see [58]).

Hence, taking into account that the Jacobian matrix $H$ associated with the complex stretching of variable $\mathbf{S}$ becomes diagonal in a particular local orthogonal system of reference, we abuse of the notation, and tensors $A, C, \tilde{A}$, and $\tilde{C}$ and also the spatial derivatives in problems (40)-(42) and (57)-(59) must be understood with respect to this particular system of reference.

Under these considerations, the coefficients of tensors $\tilde{A}$ and $\tilde{C}$ are given by (47) and (48), respectively, where now $\gamma_{1}$ and $\gamma_{2}$ are the diagonal coefficients of matrix $\Lambda$.

Finally, we recall the assumptions done for the coefficients of tensors $A$ and $C$, which appear in the original elliptic problem (40)-(42). We suppose that they are analytic functions with respect to the spatial variables in $\mathbf{S}\left(\Omega_{\mathrm{B}}\right)$ and entire (analytic in $\mathbb{C}$ ) with respect to the angular frequency $\omega$. Moreover, tensor $C$ satisfies the following coercive condition: there exists a constant $\alpha>0$ such that

$$
\operatorname{Re}\left(C_{i j k l} \psi_{i j} \bar{\psi}_{k l}\right) \geq \alpha \psi_{i j} \bar{\psi}_{i j} \quad \forall \psi \in \mathbb{C}^{2 \times 2}, \psi \neq 0
$$


uniformly for all $\mathrm{x} \in \Omega_{\mathrm{B}}$ and for all $\omega>0$.

We also require the following milder condition on tensor $-A$ : there exists an angular frequency $\omega_{0}$ and a constant $\alpha>0$ such that

$$
-\operatorname{Re}\left(A_{i j} \phi_{i} \bar{\phi}_{j}\right) \geq \alpha \phi_{i} \bar{\phi}_{i}
$$

uniformly for all $\mathrm{x} \in \Omega_{\mathrm{B}}$.

Problem (57)-(59) can be written in a weak form as follows:

Given $\omega>0$ and $\mathbf{f} \in \mathrm{L}^{2}\left(\Omega_{\mathrm{B}}\right)^{2}$ with compact support in $\Omega_{\mathrm{F}}$, find $\tilde{\mathbf{u}} \in \mathrm{H}_{\Gamma_{\mathrm{D}}}^{1}\left(\Omega_{\mathrm{B}}\right)^{2}$ such that

$$
\int_{\Omega_{\mathrm{B}}}\left(\tilde{C}_{i j k l} \tilde{u}_{i, j} \overline{\tilde{v}}_{k, l}-\tilde{A}_{i j} \tilde{u}_{j} \overline{\tilde{v}}_{i}\right) \mathrm{d} V=\int_{\Omega_{\mathrm{B}}} f_{i} \overline{\tilde{v}}_{i},
$$

for all $\tilde{\mathbf{v}} \in \mathrm{H}_{\Gamma_{\mathrm{D}}}^{1}\left(\Omega_{\mathrm{B}}\right)^{2}$.

Now, under assumptions (60)-(63), we are in a position to prove a standard existence and uniqueness result based on the analytic Fredholm's alternative theorem (see $[30])$.

Theorem 1 Let us assume conditions (60)-(63) and $\mathbf{f} \in \mathrm{L}^{2}\left(\Omega_{\mathrm{B}}\right)^{2}$ with compact support in $\Omega_{\mathrm{F}}$. If, for all $\omega \in \mathbb{C}$, there exists a constant $\beta>0$ such that

$$
\begin{aligned}
& \operatorname{Re}\left(\left(\frac{\gamma_{2}}{\gamma_{1}}-1\right) C_{i 1 j 1} \phi_{i} \bar{\phi}_{j}\right) \geq 0, \\
& \operatorname{Re}\left(\left(\frac{\gamma_{1}}{\gamma_{2}}-1\right) C_{i 2 j 2} \phi_{i} \bar{\phi}_{j}\right) \geq 0,
\end{aligned}
$$

and for $\omega_{0} \in \mathbb{C}$, fixed in (63), the inequality

$$
-\operatorname{Re}\left(\left(\gamma_{1} \gamma_{2}-1\right) A_{i j} \phi_{i} \bar{\phi}_{j}\right) \geq 0,
$$

holds then there exists a unique solution $\tilde{\mathbf{u}} \in \mathrm{H}_{\Gamma_{\mathrm{D}}}^{1}\left(\Omega_{\mathrm{B}}\right)^{2}$ of problem (64) for all $\omega \in \mathbb{C}$ except, at most, for a countable set of angular frequencies $\omega$, which can be possibly complex.

The sketch of the proof is as follows. First, since $A_{i j}$ and $C_{i j k l}$ are analytic functions and $\mathbf{S}$ satisfies (60), then $\tilde{A}_{i j}$ and $\tilde{C}_{i j k l}$ are bounded in $\Omega_{\mathrm{B}}$. This fact allows us to define the following continuous sesquilinear forms, whose domain of definition is $\mathrm{H}_{\Gamma_{\mathrm{D}}}^{1}\left(\Omega_{\mathrm{B}}\right)^{2} \times$ $\mathrm{H}_{\Gamma_{\mathrm{D}}}^{1}\left(\Omega_{\mathrm{B}}\right)^{2}$,

$$
\begin{aligned}
& a(\tilde{\mathbf{v}}, \tilde{\mathbf{w}})=\int_{\Omega_{\mathrm{B}}}\left(\tilde{C}_{i j k l} \tilde{v}_{i, j} \overline{\tilde{w}}_{k, l}+\tilde{v}_{i} \overline{\tilde{w}}_{i}\right) \mathrm{d} V \\
& b(\tilde{\mathbf{v}}, \tilde{\mathbf{w}})=\int_{\Omega_{\mathrm{B}}}\left(\tilde{A}_{i j} \tilde{v}_{i} \overline{\tilde{w}}_{j}+\tilde{v}_{i} \overline{\tilde{w}}_{i}\right) \mathrm{d} V .
\end{aligned}
$$

Now, the weak problem (64) takes the writing

$$
a(\tilde{\mathbf{u}}, \tilde{\mathbf{v}})-b(\tilde{\mathbf{u}}, \tilde{\mathbf{v}})=\int_{\Omega_{\mathrm{B}}} f \overline{\tilde{v}} \mathrm{~d} V .
$$

From (62) and (65)-(66), tensor $C$ satisfies the estimate

$$
\begin{aligned}
\operatorname{Re}\left(\tilde{C}_{i j k l} \psi_{i j} \bar{\psi}_{k l}\right)=\operatorname{Re}\left(C_{i j k l} \psi_{i j} \bar{\psi}_{k l}+\right. & \left(\frac{\gamma_{2}}{\gamma_{1}}-1\right) C_{i 1 k 1} \psi_{i 1} \bar{\psi}_{k 1} \\
& \left.+\left(\frac{\gamma_{1}}{\gamma_{2}}-1\right) C_{i 2 k 2} \psi_{i 2} \bar{\psi}_{k 2}\right) \geq \alpha \psi_{i j} \bar{\psi}_{i j}
\end{aligned}
$$


for all $\psi \in \mathbb{C}^{2 \times 2}$. Hence, the form $a(\cdot, \cdot)$ is strongly coercive and using the LaxMilgrams's lemma, we can define the invertible linear operator $T: \mathrm{L}^{2}\left(\Omega_{\mathrm{B}}\right)^{2} \rightarrow \mathrm{H}_{\Gamma_{\mathrm{D}}}^{1}\left(\Omega_{\mathrm{B}}\right)^{2}$ given by

$$
a(T \tilde{\mathbf{u}}, \tilde{\mathbf{v}})=\int_{\Omega_{\mathrm{B}}} \tilde{\mathbf{u}} \overline{\tilde{v}} \mathrm{~d} V \quad \forall \tilde{\mathbf{v}} \in \mathrm{H}_{\Gamma_{\mathrm{D}}}^{1}\left(\Omega_{\mathrm{B}}\right)^{2} .
$$

By using the Riesz representation theorem, we define the bounded linear operator associated with the form $b(\cdot, \cdot) Q: \mathrm{L}^{2}\left(\Omega_{\mathrm{B}}\right)^{2} \rightarrow \mathrm{L}^{2}\left(\Omega_{\mathrm{B}}\right)^{2}$

$$
\int_{\Omega_{\mathrm{B}}}(Q \tilde{u})_{i} \overline{\tilde{v}}_{i} \mathrm{~d} V=b(\tilde{\mathbf{u}}, \tilde{\mathbf{v}}) \quad \forall \tilde{\mathbf{v}} \in \mathrm{H}_{\Gamma_{\mathrm{D}}}^{1}\left(\Omega_{\mathrm{B}}\right)^{2} .
$$

Thus, (68) can be rewritten as $(I-Q T) \tilde{\mathbf{u}}=\mathbf{f}$ where $I$ denotes the identity operator in $\mathrm{L}^{2}\left(\Omega_{\mathrm{B}}\right)^{2}$. Finally, assumption (63), the analyticity of $\mathbf{S}$ and tensors $\tilde{A}, \tilde{C}$ with respect to $\omega$ and the compactness of operator $T$, understood as an operator with image space $\mathrm{L}^{2}\left(\Omega_{\mathrm{B}}\right)^{2}$, complete the set of hypothesis to apply the analytic Fredholm's Alternative Theorem (see [30]). To fix which alternative of the theorem is applicable, we only need to find a complex point $\omega_{0}$ such that (68) has a unique solution.

Such condition is satisfied by using the conditions (63) and (67) on tensor $A$, which imply that operator $Q$ is bounded from below (see [51]) for $\omega_{0} \in \mathbb{C}$, and the strong ellipticity of the form $a(\cdot, \cdot)$. Hence the existence and uniqueness of solution, except for a countable set of isolated complex angular frequencies $\left\{\omega_{i}\right\}_{i \in \mathbb{N}}$, is ensured. This concludes the proof of the result.

Clearly, there is a wide class of mathematical models of interest, which are not covered by the ideas developed in this section. For instance, the two-dimensional Maxwell's equations or the equations for the displacement field in linear acoustics do not satisfy assumptions (60)-(63), since operator $T$ is not invertible in $\mathrm{H}^{1}\left(\Omega_{\mathrm{B}}\right)^{2}$.

Let us remark that the proof of Theorem 1 is a simple extension of some arguments used by Collino and Monk in [28], where the existence and uniqueness of solution for the radial PML problem involving the Helmholtz equation is analyzed.

The general framework where the previous result has been proved suffers from two main lacks. First, Theorem 1 does not guarantee the existence and uniqueness of solution for all positive value of the angular frequencies what, in most of real problems, concerns the solution of the mechanical problem.

Second, the previous theoretical arguments can also provide an estimate of the norm of the solution in terms of the norm of the data, i.e., there exists a positive constant $M$ such that

$$
\int_{\Omega_{\mathrm{B}}} \tilde{u}_{i} \overline{\tilde{u}}_{i} \mathrm{~d} V \leq M \int_{\Omega_{\mathrm{B}}} f_{i} \bar{f}_{i} \mathrm{~d} V .
$$

However, it does not add any valuable information since the positive constant $M$ is neither independent nor given explicitly from the absorbing functions $\gamma_{j}$, the thickness of the PML domain, and the value of the angular frequency.

These drawbacks can be avoided if the general framework is abandoned and the PML technique is analyzed for a precise problem, and a particular choice of the system of coordinates is made. In the following subsection we review those results that complete the theoretical framework and fill the lacks found for Theorem 1 in the case of the Helmholtz equation.

Let us mention that there exist promising new approaches to the PML problem (see [34] or [52]) which analyze the spectrum of the operator $(I-Q T)$. Consequently, new norm resolvent estimates to obtain explicitly the constant $M$ in (69) could establish general PML convergence results. 
5.2 Helmholtz theoretical results

In what follows, we collect the theoretical results derived by Bramble and Pasciak (see [22]). Similar results can also be reported from Bao and $\mathrm{Wu}$ in [7]. We adapt these results to the framework used in this section.

Let us recall that the original scattering problem described in that work involves the Helmholtz equation stated in an unbounded domain where the so-called Sommerfeld radiation condition is imposed at infinity. More precisely, the statement of the problem is as follows: given $\omega>0$ and $f \in \mathrm{L}^{2}\left(\Omega_{\mathrm{B}}\right)$ with compact support in $\Omega_{\mathrm{F}}$, find $u$ such that

$$
\begin{aligned}
-\Delta u-\omega^{2} u & =f & & \text { in } \mathbb{R}^{2} \backslash \bar{O}, \\
u & =0 & & \text { on } \partial O, \\
\lim _{r \rightarrow \infty} \sqrt{r}\left(\frac{\partial u}{\partial r}-i \omega u\right) & =0 . & &
\end{aligned}
$$

We notice that the above homogeneous Dirichlet boundary condition can be replaced by a Neumann one (see [22]).

We must also remark that, even in the case of radial PML for the Helmholtz equation, as far as the authors' knowledge goes, there exist only theoretical results on convergence, existence and uniqueness of the weak solution for the PML problem (57)-(59) if the absorbing function is bounded and chosen with a specific profile.

Let us assume that the radial PML technique uses the complex stretching of variable defined by (49) where $\Omega_{\mathrm{A}}$ is placed between the circumference of radius $R_{0}$ and any smooth curve exterior to the circumference of radius $R^{\star}$. The absorbing function is assumed twice differentiable and given by

$$
\gamma_{r}(r)= \begin{cases}1 & \text { if } r \leq R_{0}, \\ 1+i \tilde{\sigma}(r) & \text { if } R_{0}<r<R_{1}, \\ 1+i \sigma_{0} & \text { if } r \geq R_{1},\end{cases}
$$

where $R_{1}<R^{\star}, \sigma_{0}$ is a positive constant and $\tilde{\sigma}$ is an arbitrary twice differentiable function, which can be explicitly written via a fifth-order polynomial (see [22] for further details).

Theorem 2 Let $\omega>0$ and $f \in \mathrm{L}^{2}\left(\Omega_{\mathrm{B}}\right)$ with compact support in $\Omega_{\mathrm{F}}$. If $R^{\star}$ is large enough and the absorbing function (73) is used, then there exists a unique solution $\tilde{u} \in \mathrm{H}^{1}\left(\Omega_{\mathrm{B}}\right)$ ) of the PML problem (57)-(59) associated with the Helmholtz problem (70)-(72). Moreover, there exist a positive constant $M$ independent of $\sigma_{0}$ and $R^{\star}$ such that

$$
\|\tilde{u}-u\|_{\mathrm{L}^{2}\left(\Omega_{\mathrm{F}}\right)} \leq M e^{-2 \sigma_{0} \omega R^{\star}}\|f\|_{\mathrm{L}^{2}\left(\Omega_{\mathrm{B}}\right)} .
$$

Although this exponential estimate can be extended to other models as the Maxwell equations [22], all the theoretical analysis has been done for the radial PML technique and, in fact, the provided arguments cannot be straightforwardly extended to the Cartesian PML technique.

Consequently, until now it does not exist any mathematical support, even for the Helmholtz equation, to ensure the existence and uniqueness of solution of the Cartesian PML problem for all positive angular frequencies and, moreover, there is not any general 
result on the error introduced by the PML problem with respect to the solution of the original scattering problem when the thickness of the PML domain $\Omega_{\mathrm{A}}$ is enlarged or the modulus of $\gamma_{j}, j=1,2$ are increased.

\section{Optimal PML for the Helmholtz equation}

We have already derived the construction of the PML equation by means of a complex stretching of coordinates. We have also described the PML technique for problems involving second order partial differential equations. However, in general (except partially [56]) theoretical results about existence, uniqueness and error estimates does not take advantage of the knowledge of this derivation. Indeed, we have also failed to obtain general results for the existence and uniqueness of solution for all $\omega>0$.

We have already claimed that the absorbing functions play a key role in the damping behavior of the PML equations, and thereby, in the accuracy and robustness of the discrete method Since we have checked that the real and imaginary parts of the absorbing functions must be settled adequately for each particular model, trying to determine a universal optimal absorbing function makes nonsense.

However, if the model is fixed then it is possible to find a kind of optimal absorbing functions, which theoretically recover the exact solution of the unbounded problem using a PML domain of finite thickness. Thus, in the sequel, we describe such optimality features of singular absorbing functions in the case of Helmholtz equation.

In the PML literature, most absorbing functions considered for Helmholtz equation keep the physical expression inherited by the time dependent formulation of the same model, and hence absorbing functions are given by expressions of the type $1+i \sigma /(\rho \omega)$, (already introduced in Section 2), where $\sigma$ is assumed to be non-negative, continuous inside the PML and monotonically increasing.

Throughout the rest of this section, we only consider absorbing functions as described above and, moreover, despite the assumptions done on the regularity of $\sigma$ in Theorem 2, no requirements are imposed for its values on $\Gamma_{\mathrm{I}}$. Hence, the null condition $\sigma=0$ on the interface between the physical domain and the PML is not required for the optimal absorbing functions.

Fixed the PML domain, the accuracy of standard PML techniques for the Helmholtz problem relies on taking absorbing functions with bounded imaginary part and such that their integrals are large enough. Until recently, as far as the knowledge of the authors goes, there are few works (precisely, we should remark those from [22], [58] and [34]), providing valuable theoretical results that confirms this numerical evidence for arbitrary variable profiles of $\sigma$.

We propose, instead (see [17]), to use an unbounded profile for $\sigma$ such that the integral through a transverse section of the PML domain is infinite.

Throughout the rest of this section, first we compare the exact results of bounded and singular absorbing functions for the one-dimensional Helmholtz equation derived from a plane wave analysis at oblique incidence. More precisely, if the PML domain is placed in the interval $\left(L, L^{\star}\right)$, then we compare the most widely used classical choice, namely the quadratic function taking the value $\sigma^{\star}$ on the exterior boundary of the PML domain,

$$
\sigma(x)=\sigma^{(\mathrm{Q})}(s)=\frac{\sigma^{\star}}{\left(L^{\star}-L\right)^{2}}(x-L)^{2}, \quad x \in\left(L, L^{\star}\right),
$$


and the following singular function with unbounded integral in $\left(L, L^{\star}\right)$,

$$
\sigma(x)=\sigma^{(\mathrm{U})}(x)=\frac{c}{L^{\star}-x}, \quad x \in\left(L, L^{\star}\right) .
$$

Obviously, other kind of absorbing functions, as linear or constant functions can be considered (see, for instance, $[10,13,28]$ ), and also other kind of non-integrable functions can be taken into account.

Moreover, we could wonder whether among this wide class of non-integrable functions, there is one which is optimal. To the best of our knowledge, there are not a precise answer and we can only compare different choices of non-integrable absorbing functions. A detailed comparison of different singular absorbing functions is described in [17].

Until now, there are not theoretical results guaranteeing the use of such kind of singular absorbing functions for the Cartesian PML. However, we show in the next subsections numerical evidences of the good accuracy in a simple plane wave analysis and also in two-dimensional numerical simulations. The theoretical proof of the exactness of Cartesian PML with non-integral absorbing functions is currently an open problem.

6.1 Plane wave analysis of the Cartesian PML

We illustrate the effect of using non-integrable absorbing functions in the behavior of the PML technique for a one-dimensional toy problem, where the exact solution has a closed form.

We recover a one-dimensional model that comes from the propagation of plane pressure waves with an oblique angle of incidence $\theta$. Notice that a similar model (see (21)-(23)) has been already used to illustrate the ideas introduced in Section 3. Let us write the equations describing the following one-dimensional problem stated in the unbounded interval $(0, \infty)$ :

Find $u$ such that

$$
\begin{aligned}
-u^{\prime \prime}-k^{2} \cos ^{2}(\theta) u=0 & \text { in }(0,+\infty), \\
u^{\prime}+i k \cos (\theta) u=2 i k \cos (\theta) & \text { at } x=0, \\
u^{\prime}-i k \cos (\theta) u=0 & \text { at } x \rightarrow+\infty,
\end{aligned}
$$

where $k=\omega / c$ is the wavenumber, expressed in terms of the angular frequency $\omega$ and the sound speed of the fluid $c$. Notice that the mass density has been simplified from (76)-(78) by the sake of simplicity and, in what follows, it will be assumed equal to $1 \mathrm{~kg} / \mathrm{m}^{3}$.

We introduce a PML in the interval $\left(L, L^{\star}\right)$ to truncate the unbounded interval $(0,+\infty)$. As we have already mentioned, we use a variable profile for the absorbing function of the type

$$
\gamma(x)= \begin{cases}1 & \text { in }(0, L), \\ 1+i \frac{\sigma(x)}{\omega} & \text { in }\left[L, L^{\star}\right)\end{cases}
$$

where we recall that $\sigma$ is taken to be a quadratic or singular profile. 
Taking into account that the PML equation is derived by using a complex change of variable and that the solution of the original problem is analytic, it is clear that the solution of the PML problem can be written as a superposition of plane waves:

$$
\tilde{u}(x)=A e^{i k \cos (\theta) \tilde{x}(x)}+B e^{-i k \cos (\theta) \tilde{x}(x)}, \quad x \in\left(0, L^{\star}\right),
$$

where $A$ is the amplitude of the incident wave incoming from the boundary $x=0, B$ that of the reflected wave by the absorbing layer and $\tilde{x}$ defines the complex stretching of the spatial variable as

$$
\tilde{x}(x)= \begin{cases}x & \text { in }(0, L), \\ L+\int_{L}^{x} \gamma(s) \mathrm{d} s & \text { in }\left[L, L^{\star}\right) .\end{cases}
$$

Hence, from the boundary condition at $x=0$ and the homogeneous Dirichlet boundary condition at $x=L^{\star}$, we obtain

$$
A=1-B, \quad B=\frac{e^{2 i k \cos (\theta) L^{\star}}}{e^{2 i k \cos (\theta) L^{\star}}-e^{\frac{2 \cos (\theta)}{c} \int_{L}^{L^{\star}} \sigma(s) \mathrm{d} s} .}
$$

Notice that the term involving $B$ arises as a consequence of the waves reflected at $x=L^{\star}$. Expression (79) for $B$ shows that the larger the integral $\int_{L}^{L^{\star}} \sigma(s) \mathrm{d} s$, the closer the $B$ to zero and, consequently, the closer the $\tilde{u}$ to the solution $u$ of original problem $(76)-(78)$ in the physical domain $(0, L)$. Indeed, straightforward computations lead to

$$
\int_{0}^{L}|\tilde{u}(s)-u(s)|^{2} \mathrm{~d} s=|B|^{2} \frac{2 k \cos (\theta) L-\sin (2 k \cos (\theta) L)}{k \cos (\theta)} .
$$

It is clear that if we use a non-integrable function, as $\sigma^{(\mathrm{U})}$ defined in (75), then $B=0$ and, consequently, the resulting $\tilde{u}$ will coincide exactly with the solution $u$ of problem (76)-(78) in the physical domain.

In order to illustrate this behavior we take the following parameters: $L=0.5 \mathrm{~m}$, $L^{\star}=0.6 \mathrm{~m}, \omega=1200 \mathrm{rad} / \mathrm{s}, c=340 \mathrm{~m} / \mathrm{s}$, and $\theta=\frac{3 \pi}{8} \mathrm{rad}$. We compare two examples of the above mentioned absorbing functions: a classical quadratic absorbing function, namely $\sigma^{(\mathrm{Q})}$ defined in (74), and the non-integrable function $\sigma^{(\mathrm{U})}$.

In the plots of Figure 11 we can see that, when choosing the quadratic function, $\tilde{u}$ approximates the exact solution $u$ as $\sigma^{\star}$ becomes large. The reflection coefficient is, in this case, $B=0.26$ for $\sigma^{\star}=50 c$, and $B=2.88 \times 10^{-6}$ for $\sigma^{\star}=500 c$. In the same figure we can see that the error is null when choosing the unbounded absorbing function, $\sigma^{(\mathrm{U})}$.

In Figure 12 we show the dependence of $B$ with respect to the thickness of the PML and to the value of $\sigma^{\star}$, when $\sigma^{(\mathrm{Q})}$ is used. As it is expected, in both cases the spurious reflection $B$ decreases when the thickness or the value of $\sigma^{\star}$ increases.

In the left-hand plot of Figure 13, we show the dependence of the reflection coefficient $B$ with respect to the angle of incidence of the plane wave when $\sigma^{(\mathrm{Q})}$ is used. It is important to emphasize that, in this case, the reflection coefficient (and hence the error) increases as the angle of incidence increases, whereas the error is null for any angle of incidence when $\sigma^{(\mathrm{U})}$ is used.

Analogously, in the right-hand plot of Figure 13, we show the dependence of $B$ with respect to angular frequency $\omega$ when $\sigma^{(\mathrm{Q})}$ is used. For this test we have taken again $\theta=\frac{3 \pi}{8} \mathrm{rad}$ as angle of incidence. We observe that $|B|$ achieves periodically maximum 

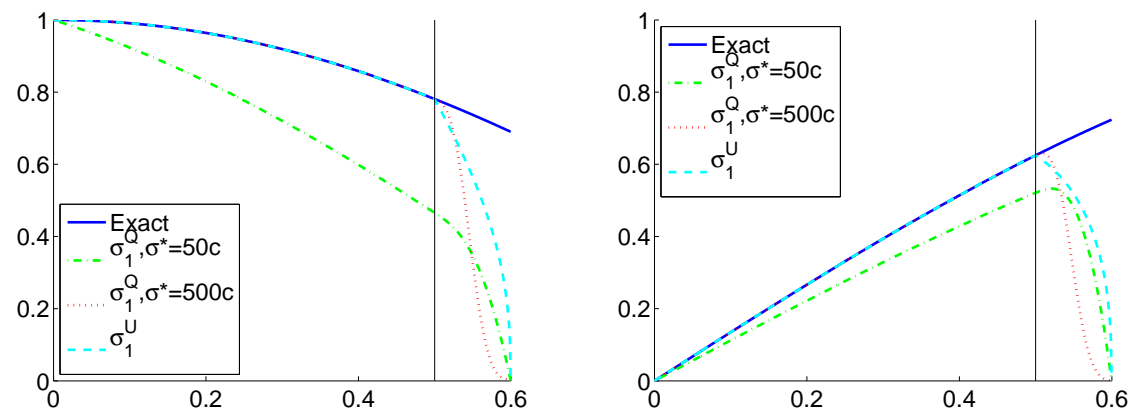

Fig. 11 Real part (left) and imaginary part (right) of exact and approximated pressures for $\theta=3 \pi / 8 \mathrm{rad}$.
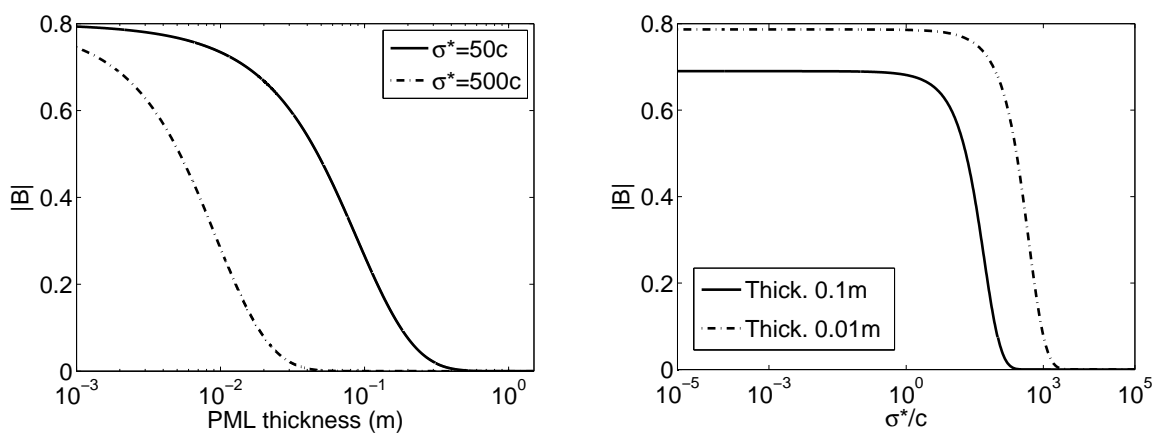

Fig. 12 Modulus of the reflection coefficient for $\sigma^{(\mathrm{Q})}$ versus the thickness of the PML (right) and $\sigma^{\star} / c$ (left).
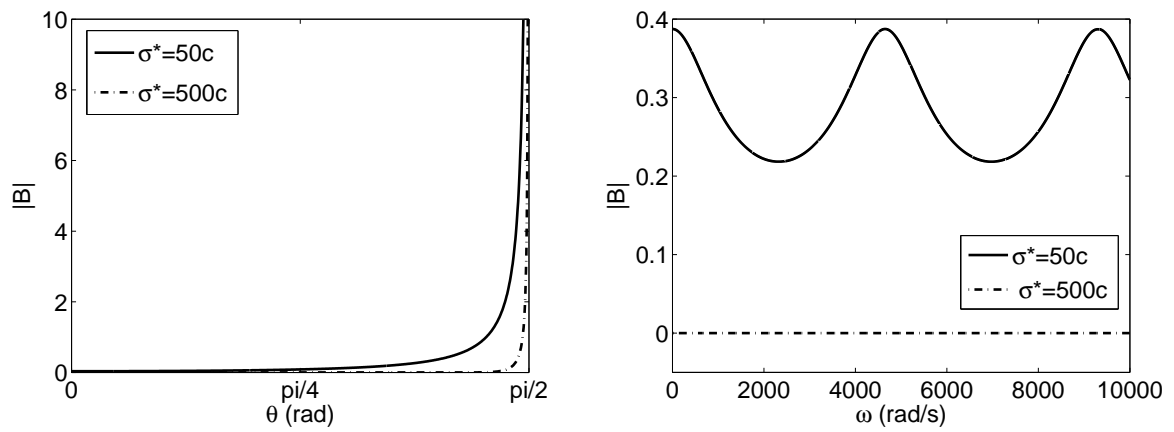

Fig. 13 Modulus of the reflection coefficient for $\sigma^{(\mathrm{Q})}$ versus incidence angle (right) and versus frequency (left).

values for certain frequencies. Again, we want to remark that taking the unbounded absorbing function $\sigma^{(\mathrm{U})}$ in the PML we recover $B=0$, for any value of the frequency. 
In summary, from this simple illustration we can draw some conclusions. We have seen that there exist two classical alternatives to obtain accurate numerical results for the PML technique: either to enlarge the thickness of the PML domain or to increase the maximum value for $\sigma$.

In the first alternative, enlarging the PML domain increases the computational cost of the PML technique. However, the spurious reflections are uniformly bounded because the exponential decay in Lemma 1 (see Section 3) remains constant. Similar numerical behavior is also achieved in two-dimensional problems.

In the second alternative, we fix the thickness of the PML domain and increase the value of $\sigma$. Obviously, if it is naively increased by taking large values for $\sigma$ in the whole PML domain, we reduce the magnitude of the spurious reflections generated by the exterior boundary of the PML domain, but, from the numerical point of view, a boundary layer is expected in the interface between the physical and PML domains.

In conclusion, the first alternative is not admissible since the computational domain remains too large to deal with real-life numerical simulations. As naive as it can look, a modification of the second alternative has not been studied until recent years (see $[17])$.

More precisely, in the next subsections we check that a non-integrable absorbing function is a good candidate to minimize the spurious reflections in the two-dimensional case. Moreover, for the radial PML technique we prove that the exact solution of the original problem is recovered even using a PML domain of finite thickness.

6.2 Numerical accuracy of Cartesian PML for the Helmholtz equation

First, let us recall the scattering problem involving the Helmholtz equation. As a particular case of problem (40)-(42), our goal is to compute an approximation with the PML technique of the following problem with homogeneous Neumann boundary data:

$$
\begin{array}{rlrl}
-\Delta u-\frac{\omega^{2}}{c^{2}} u & =f & & \text { in } \mathbb{R}^{2} \backslash \bar{O}, \\
\frac{\partial u}{\partial \mathbf{n}} & =0 & & \text { on } \partial O, \\
\lim _{r \rightarrow \infty} \sqrt{r}\left(\frac{\partial u}{\partial r}-i \omega u\right) & =0, &
\end{array}
$$

where $\mathbf{n}$ is the unit normal vector to $\partial O$ toward the exterior of $O$. We also adapt the general weak problem (64) to the particular case of the Helmholtz equation. Since now the absorbing functions are going to be non-integrable, we only require the scalar solution $\tilde{u}$ to belong to

$$
\tilde{\mathrm{H}}^{1}\left(\Omega_{\mathrm{B}}\right)=\left\{u: \Omega_{\mathrm{B}} \rightarrow \mathbb{C}:\left.u\right|_{K} \in \mathrm{H}^{1}(K), \forall K \text { compact, } K \subset \bar{\Omega}_{\mathrm{B}}, K \cap \Gamma_{\mathrm{D}}=\emptyset\right\}
$$

In fact, the weak problem is described as follows:

Given $\omega>0$ and $f \in \mathrm{L}^{2}\left(\Omega_{\mathrm{B}}\right)$ with compact support in $\Omega_{\mathrm{F}}$, find $\tilde{u} \in \tilde{\mathrm{H}}^{1}\left(\Omega_{\mathrm{B}}\right)$ such that $\tilde{u}=0$ on $\Gamma_{\mathrm{D}}$ and satisfies

$$
\int_{\Omega_{\mathrm{B}}} \frac{\gamma_{2}}{\gamma_{1}} \frac{\partial \tilde{u}}{\partial x_{1}} \frac{\partial \overline{\tilde{v}}}{\partial x_{1}} \mathrm{~d} V+\int_{\Omega_{\mathrm{B}}} \frac{\gamma_{1}}{\gamma_{2}} \frac{\partial \tilde{u}}{\partial x_{2}} \frac{\partial \overline{\tilde{v}}}{\partial x_{2}} \mathrm{~d} V-\frac{\omega^{2}}{c^{2}} \int_{\Omega_{\mathrm{B}}} \gamma_{1} \gamma_{2} \tilde{u} \tilde{\tilde{v}} \mathrm{~d} V=\int_{\Omega_{\mathrm{F}}} f \bar{v} \mathrm{~d} V
$$

for all $\tilde{v} \in \tilde{H}^{1}\left(\Omega_{\mathrm{B}}\right)$ vanishing on $\Gamma_{\mathrm{D}}$. 
As we have already introduced in the previous sections, $\gamma_{j}\left(x_{j}\right)=1$ in $\Omega_{\mathrm{F}}$ and $\gamma_{j}\left(x_{j}\right)=1+i / \omega \sigma\left(x_{j}\right)$ for $j=1,2$, where $\sigma$ can be given by (74) or (75).

Let us remark that it does not exist any theoretical support to guarantee the existence and uniqueness of solution. However, the accuracy of the numerical results and the theoretical results shown for the radial PML technique can be considered promising signs for the well-posedness of the previous weak problem.

Now we are in a position to use a standard finite element method for the numerical solution of weak problem (84) and to show that the resulting discrete problem is well posed only for certain unbounded absorbing functions. This will lead to additional constraints on $\gamma_{1}$ and $\gamma_{2}$.

We consider a triangular mesh of the physical domain $\Omega_{\mathrm{F}}$ and a rectangular mesh of the absorbing layer $\Omega_{\mathrm{A}}$, matching on the common interface between the physical and absorbing domains as shown in Figure 14. As usual, $h$ denotes the mesh-size.

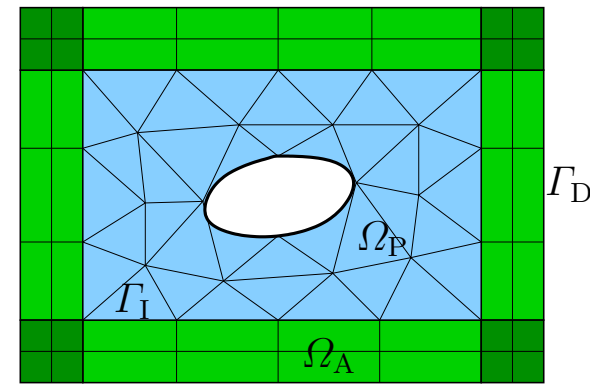

Fig. 14 Hybrid mesh on PML and physical domain.

The reason why we use such special hybrid meshes in this subsection is that triangles are more adequate to fit the boundary of the obstacle, whereas rectangles will allow us to compute explicitly the integrals involving the absorbing function appearing in the elements in the layer. This is not strictly necessary, since these integrals can also be efficiently computed by means of standard quadrature rules for triangles (see [17]). However, we will mainly consider exact integration to be able to assess the accuracy of the proposed PML independently of quadrature errors.

We compute the finite element approximations $\tilde{u}_{h}$ of the pressure field in the physical domain and in the absorbing layer, by using linear triangular finite elements for the former and bilinear rectangular finite elements for the latter. The degrees of freedom defining the finite element solution are the values of $\tilde{u}_{h}$ at the vertices of the elements. Notice that, since a compatible mesh is used, each element is contained either in the physical or in the absorbing domain which ensures the continuity of $\tilde{u}_{h}$ at the vertices on the interface between $\Omega_{\mathrm{F}}$ and $\Omega_{\mathrm{A}}$.

Moreover, we impose the Dirichlet boundary condition $\tilde{u}_{h}=0$ to the finite element solution on the exterior boundary of the PML. Hence, $\tilde{u}_{h}$ does not have any degree of freedom on the outer boundary. This fact will be essential for the resulting discrete problem to be well posed when a non-integrable absorbing function is used. Indeed, computations show that the coefficients of the finite element matrices are bounded in spite of the singularity of the absorbing function on the outer boundary of the PML domain. For a detailed proof, see [17]. 
As we have seen in the previous subsection, when quadratic absorbing functions are used, the standard procedure to minimize the spurious reflections produced at the outer boundary of the PML consists in taking large values for $\sigma^{\star}$. However, larger values of $\sigma^{\star}$ lead to larger discretization errors. Therefore, $\sigma^{\star}$ cannot be chosen arbitrarily large because, otherwise, the discretization errors would be dominant, deteriorating the overall accuracy of the method.

As shown in [27], for a given problem and a given mesh there is an optimal value of $\sigma^{\star}$ leading to minimal errors. Unfortunately, such optimal value depends strongly on the problem data as well as on the particular mesh. Thus, in practice, it is necessary to find a reasonable value of $\sigma^{\star}$ in advance. No theoretical procedure to tune this parameter is known to date. Some efforts have been done in [74], but the dependence of $\sigma^{*}$ with respect to the mesh has not been avoided.

With the purpose of comparison, we have applied our PML method with a nonintegrable absorbing profile $\sigma$ to a scattering problem with known analytical solution and assessed the accuracy of the numerical results.

Consider problem (81)-(83) where the obstacle $O$ is the unit circle centered at the origin. Given any inner point $\left(x_{1}^{0}, x_{2}^{0}\right)$ of this circle, it is well known that the function

$$
u(\mathbf{x})=\frac{i}{4} \mathrm{H}_{0}^{(1)}\left(k \sqrt{\left(x_{1}-x_{1}^{0}\right)^{2}+\left(x_{2}-x_{2}^{0}\right)^{2}}\right)
$$

satisfies (81) and (83). Therefore, if the Neumann condition is adequately chosen then $u$ is the unique solution to this problem.

In our experiments we have taken $x_{1}^{0}=0.5 \mathrm{~m}, x_{2}^{0}=0, c=340 \mathrm{~m} / \mathrm{s}$, and different values of angular frequency $\omega$. As computational domains we have taken $\Omega_{\mathrm{B}}=$ $\left(-L^{\star}, L^{\star}\right) \times\left(-L^{\star}, L^{\star}\right)$ and $\Omega_{\mathrm{B}}=(-L, L) \times(-L, L)$ with $L^{\star}=2.25 \mathrm{~m}$ and $L=2.0 \mathrm{~m}$ (see Figure 15).

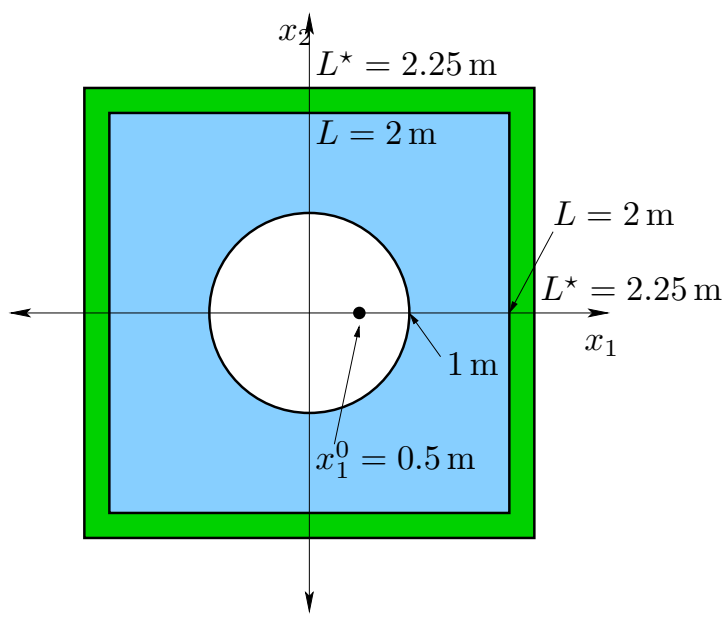

Fig. 15 Domain of the scattering problem.

We have used uniform refinements of the mesh shown in Figure 15; the number $N$ of elements through the thickness of the PML is used to label each mesh. 
To measure the accuracy we have estimated the relative error in the $\mathrm{L}^{2}$-norm in $\Omega_{\mathrm{F}}$ by:

$$
\text { Error }:=\frac{\left\|\tilde{u}_{h}-\Pi_{h} u\right\|_{\mathrm{L}^{2}\left(\Omega_{\mathrm{F}}\right)}}{\left\|\Pi_{h} u\right\|_{\mathrm{L}^{2}\left(\Omega_{\mathrm{F}}\right)}}
$$

where we recall that $\tilde{u}_{h}$ is the numerical solution and $\Pi_{h} u$ is the Lagrange interpolant of the exact solution $u$.

In Table 1 we compare the errors of the PML method obtained by using the unbounded absorbing functions (75) and the quadratic absorbing functions (74). For the latter, we have used the optimal value of $\sigma^{\star}$, which is also reported in the table. We also include the condition number $\kappa$ of the system matrix for each discrete problem.

Let us remark that, in both cases, we recover an order two of convergence in the $\mathrm{L}^{2}$-norm, which is optimal for the proposed finite element discretization.

Table 1 Comparison of PML methods with unbounded and quadratic absorbing functions.

\begin{tabular}{|c|c|c|c|c|c|c|}
\hline \multirow[b]{2}{*}{$\omega(\mathrm{rad} / \mathrm{s})$} & \multirow[b]{2}{*}{ Mesh } & \multicolumn{2}{|c|}{ Unbounded (75) } & \multicolumn{3}{|c|}{ Quadratic (74) } \\
\hline & & Error(\%) & $\kappa$ & $\sigma^{\star}$ & Error(\%) & $\kappa$ \\
\hline \multirow{3}{*}{250} & $N=2$ & 0.763 & $6.7 \mathrm{e}+02$ & $22.28 c$ & 11.644 & $4.7 \mathrm{e}+02$ \\
\hline & $N=4$ & 0.131 & $5.1 \mathrm{e}+03$ & $29.57 c$ & 3.675 & $5.0 \mathrm{e}+03$ \\
\hline & $N=8$ & 0.029 & $4.1 \mathrm{e}+04$ & $38.37 c$ & 1.134 & $4.6 \mathrm{e}+04$ \\
\hline \multirow{3}{*}{750} & $N=2$ & 1.700 & $1.1 \mathrm{e}+02$ & $27.67 c$ & 7.602 & $1.1 \mathrm{e}+02$ \\
\hline & $N=4$ & 0.447 & $7.0 \mathrm{e}+02$ & $35.52 c$ & 2.291 & $9.4 \mathrm{e}+02$ \\
\hline & $N=8$ & 0.109 & $5.6 \mathrm{e}+03$ & $43.49 c$ & 0.698 & $8.2 \mathrm{e}+03$ \\
\hline \multirow{3}{*}{1250} & $N=2$ & 6.958 & $2.7 \mathrm{e}+02$ & $27.89 c$ & 11.620 & $2.9 \mathrm{e}+02$ \\
\hline & $N=4$ & 1.946 & $1.1 \mathrm{e}+03$ & $36.94 c$ & 3.336 & $1.7 \mathrm{e}+03$ \\
\hline & $N=8$ & 0.430 & $9.7 \mathrm{e}+03$ & $45.70 c$ & 0.919 & $1.5 \mathrm{e}+03$ \\
\hline
\end{tabular}

A significant advantage of the proposed unbounded absorbing functions (75) can be clearly appreciated from this table. This is particularly remarkable for the lowest frequencies, but the errors with the quadratic absorbing functions are larger in all cases, even though the optimal value of $\sigma^{\star}$ has been used. On the other hand, in spite of the singular character of the unbounded functions, the condition numbers of the resulting system matrices are essentially of the same order of magnitude as those of the quadratic functions.

On the other hand, Table 1 also shows that the optimal value of $\sigma^{\star}$ strongly depends on the problem data (the frequency $\omega$ in this case) and the mesh. The errors and the condition numbers $\kappa$ would be significantly larger if any other value than the optimal $\sigma^{\star}$ were used.

As a conclusion, the proposed PML method with unbounded absorbing function (75) clearly beats the classical choice of bounded absorbing functions. Moreover, it overcomes the problem of determining optimal parameters.

Similar numerical results can be found by using numerical quadrature rules with inner nodes not only on quadrilateral meshes (see [17]) but also on triangular meshes (see [66]). 
6.3 Optimal radial PML for Helmholtz equation

Radial PML methods are based on simulating dissipation in an annular domain, $\Omega_{\mathrm{A}}=$ $\left\{\mathbf{x} \in \mathbb{R}^{2}: R<|\mathbf{x}|<R^{\star}\right\}$, surrounding the physical domain of interest $\Omega_{\mathrm{F}}$. The first contribution to the construction of the radial PML equation was done by Collino and Monk [28] who, by means of a complex-valued radial stretching, wrote the radial PML governing equation for the Helmholtz equation. This construction is equal to that done in Subsection 4.3 for general second order problems.

If we apply the radial PML technique with singular absorbing functions to the Helmholtz problem (70)-(72), the weak problem is written as follows:

Given $\omega>0$ and $f \in \mathrm{L}^{2}\left(\Omega_{\mathrm{B}}\right)$ with compact support in $\Omega_{\mathrm{F}}$, find $\tilde{u} \in \tilde{\mathrm{H}}^{1}\left(\Omega_{\mathrm{B}}\right)$ such that $\tilde{u}=0$ on $\partial O$ and satisfies

$$
\int_{\Omega_{\mathrm{B}}} \operatorname{det}(H) H^{-1} H^{-t} \operatorname{grad} \tilde{u} \cdot \overline{\tilde{v}} \mathrm{~d} V-\frac{\omega^{2}}{c^{2}} \int_{\Omega_{\mathrm{B}}} \operatorname{det}(H) \tilde{u} \overline{\tilde{v}} \mathrm{~d} V=\int_{\Omega_{\mathrm{F}}} f \overline{\tilde{v}} \mathrm{~d} V,
$$

for all $\tilde{v} \in \tilde{\mathrm{H}}^{1}\left(\Omega_{\mathrm{B}}\right)$ vanishing on $\partial O$.

Notice that, as we have introduced in Section 4, matrix $H$ is given by

$$
H=\left(\begin{array}{cc}
\cos \theta & \sin \theta \\
-\sin \theta & \cos \theta
\end{array}\right)^{t}\left(\begin{array}{cc}
\gamma_{r} & 0 \\
0 & \tilde{\gamma}
\end{array}\right)\left(\begin{array}{cc}
\cos \theta & \sin \theta \\
-\sin \theta & \cos \theta
\end{array}\right)
$$

where we recall that $\tilde{\gamma}=\frac{1}{r}\left(R+\int_{R}^{r} \gamma_{r}(s) d s\right)$ and $\gamma_{r}$ is a complex-valued function satisfying $\gamma_{r}=1$ in $\Omega_{\mathrm{F}}$ and $\gamma_{r}(r)=1+(i / \omega) \sigma(r)$ in $\Omega_{\mathrm{A}}$, being $\sigma$ a positive nondecreasing function.

In the development of [66], we have extended the previous existence and uniqueness results to the case of a non-integrable absorbing function (see also [19] for details). In order to prove the existence and uniqueness of solution to the weak PML problem (85) when a non-integrable function is used, we have to introduce the non-standard weighted Sobolev space

$$
\begin{aligned}
\mathrm{V}:=\left\{\tilde{v} \in \tilde{\mathrm{H}}^{1}\left(\Omega_{\mathrm{B}}\right):\|\tilde{v}\|_{\mathrm{V}}^{2}=\int_{\Omega_{\mathrm{B}}} \operatorname{det}(J) J^{-1} J^{-t} \operatorname{grad} \tilde{v} \cdot \operatorname{grad} \overline{\tilde{v}} \mathrm{~d} V\right. & \\
& \left.\quad+\int_{\Omega_{\mathrm{B}}} \operatorname{det}(J)|\tilde{v}|^{2} \mathrm{~d} V<+\infty\right\},
\end{aligned}
$$

where $J$ is the matrix given by

$$
J=\left(\begin{array}{cc}
\cos \theta & \sin \theta \\
-\sin \theta & \cos \theta
\end{array}\right)^{t}\left(\begin{array}{cc}
\left|\gamma_{r}\right| & 0 \\
0 & |\tilde{\gamma}|
\end{array}\right)\left(\begin{array}{cc}
\cos \theta & \sin \theta \\
-\sin \theta & \cos \theta
\end{array}\right)
$$

Matrix $J$ is closely related to matrix $H$ introduced in Section 4 . In fact, $J$ is obtained by replacing the diagonal coefficients of $H$ by their modulus.

We have proved in [19] that the solution of the weak problem (85) is equal to the solution of the original problem (70)-(72), if function $\sigma$ is not integrable and with the unique restriction of belonging to the Holder space $\mathcal{C}^{1,2}$ locally in the PML domain.

Theorem 3 Let $\omega>0$ and $f \in \mathrm{L}^{2}\left(\Omega_{\mathrm{B}}\right)$ with compact support in $\Omega_{\mathrm{F}}$. There exists a unique solution $\tilde{u} \in \mathrm{V}$ of the weak problem (85). Moreover, $\tilde{u}$ coincides with $u$, the solution of the original Helmholtz problem (70)-(72), in $\Omega_{\mathrm{F}}$. 
Let us remark that the homogeneous Dirichlet boundary condition on the outer boundary of the PML domain $\Omega_{\mathrm{A}}$ is implicitly contained in the definition of the space $\mathrm{V}$. This boundary condition will be actually used in the discretization of the weak problem associated with the coupled problem.

From the numerical point of view, we can introduce a standard finite element discretization of the weak problem associated with (85). To evaluate the numerical accuracy of the radial PML technique with singular absorbing functions, we use meshes $\mathcal{T}_{h}$ of curved elements corresponding to standard quadrilaterals in polar coordinates. As usual, $h$ denotes the mesh-size.

Each element must be completely contained either in $\bar{\Omega}_{\mathrm{F}}$ or in $\bar{\Omega}_{\mathrm{A}}$. Moreover, we take advantage of the fact that $\Omega_{\mathrm{A}}$ is an annular domain by using curved rectangles in $\Omega_{\mathrm{A}}$. We use isoparametric bilinear elements in polar coordinates.

As a consequence of the definition of the weighted Sobolev space V, the homogeneous Dirichlet boundary condition on the outer boundary of the PML domain $\Omega_{\mathrm{A}}$ must be included in the definition of the finite discretization space to ensure that each discrete test function belongs to V. For the non-integrable profile

$$
\sigma(r)=\sigma^{(\mathrm{U})}(r)=\frac{c}{R^{\star}-r},
$$

being $c$ the sound speed in $\Omega_{\mathrm{F}}$, this boundary condition is also sufficient (see [18] for other feasible choices of $\sigma$ ).

In what follows we report some numerical results obtained with a computer code implementing the PML technique with a non-integrable absorbing function $\sigma^{(\mathrm{U})}$.

To illustrate the performance of the PML method with the non-integrable $\sigma^{(\mathrm{U})}$, we consider again a simple problem with a known analytical solution. It is well known that the fundamental solution of the Helmholtz problem is

$$
u(\mathbf{x})=\frac{i}{4} \mathrm{H}_{0}^{(1)}\left(\frac{\omega}{c}|\mathbf{x}|\right) .
$$

Therefore, if we take the appropriate Dirichlet data, then it should be expected that the finite element solution $\tilde{u}_{h}$ for weak problem (85) approximates $u$ in the physical domain $\Omega_{\mathrm{F}}$.

In this numerical experiment we have taken $c=340 \mathrm{~m} / \mathrm{s}$ and $\omega=750 \mathrm{rad} / \mathrm{s}$. We have considered a circular obstacle of radius $R_{0}=1 \mathrm{~m}$ (see Figure 16) and the PML layer is placed between the circumferences of radius $R=2 \mathrm{~m}$ and $R^{\star}=2.1 \mathrm{~m}$.

Because of the boundary condition $\tilde{u}=0$ on the outer boundary of the PML, all the integrals involved in the finite element method are finite (see [18] for details). To evaluate these integrals, we have used a Simpson adaptive rule to reduce the effect of the numerical errors arising from quadrature as much as possible. However, it is shown in [18] that standard quadrature rules lead to numerical results essentially of the same accuracy.

We have used uniform refinements of the mesh shown in Figure 16; the number $N$ of elements through the thickness of the PML is used to label each mesh. Specifically, meshes corresponding to $N=2,4$ and 8 have 264, 1008 and 3936 degrees of freedom, respectively.

In Figure 17 we show the real and imaginary parts of the solution computed for the PML problem with the mesh corresponding to $N=8$. The solution is plotted in the physical domain and in the PML as well. 

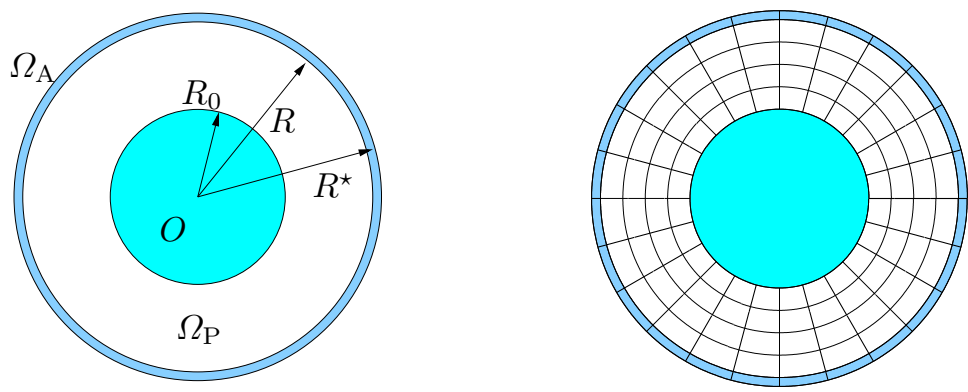

Fig. 16 Domains and mesh $(N=1)$ in the scattering problem.
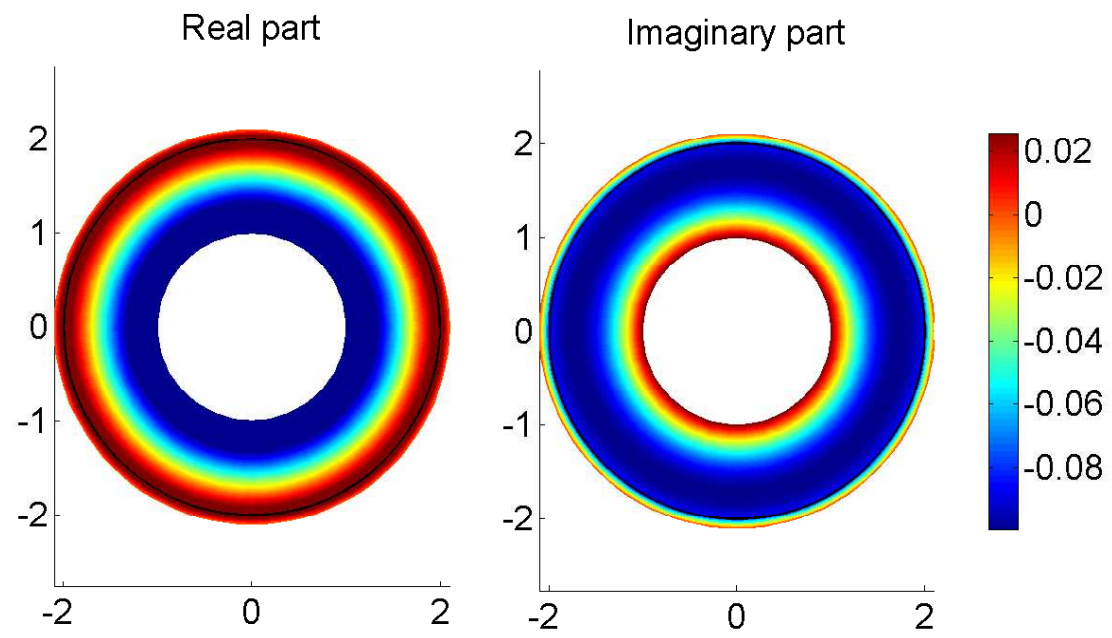

Fig. 17 Solution of the PML problem with a non-integrable absorbing function. Mesh $N=8$

Analogous to the discrete problem involving the Helmholtz equation in a bounded domain, an order of convergence $\mathcal{O}\left(h^{2}\right)$ is achieved for the $\mathrm{L}^{2}$-relative error. Let us recall that this is the optimal order for the used finite elements in $\mathrm{L}^{2}$-norm.

To end this section, we compare the numerical performance of this PML technique with that of a classical one based on the quadratic function (see for instance [13] or $[28])$ :

$$
\sigma^{(\mathrm{Q})}(s)=c \sigma^{\star}(s-R)^{2}
$$

As shown in [27], for a given problem and a given mesh there is an optimal value of $\sigma^{\star}$ leading to minimal errors. Such optimal value depends strongly on the problem data as well as on the particular mesh. Thus, in practice, it is necessary to tune it in advance. No theoretical procedure for such a tuning is known to date. 
In Table 2, we compare the errors of the two PML methods with the unbounded absorbing function (87) and with the quadratic absorbing function (88), respectively, applied to the same test problem as above. For the quadratic absorbing function, we have used the optimally tuned value of $\sigma^{\star}$, which is also reported in the table (one can see that it changes significantly from one mesh to another).

Table 2 Comparison of PML methods with non-integrable and quadratic absorbing functions.

\begin{tabular}{lcccc}
\hline & Non-integrable $\sigma^{(\mathrm{U})}(87)$ & & \multicolumn{2}{c}{ Quadratic $\sigma^{(\mathrm{Q})}(88)$} \\
\cline { 2 - 2 } \cline { 5 - 5 } Mesh & Error(\%) & & Error(\%) & $\sigma^{\star}$ \\
\hline$N=2$ & 0.6377 & & 13.7867 & 6156.45 \\
$N=4$ & 0.1558 & & 3.7986 & 8484.28 \\
$N=8$ & 0.0386 & & 1.1373 & 10712.08 \\
\hline
\end{tabular}

Table 2 shows that the errors of the PML method with the non-integrable absorbing function are significantly smaller than those of the classical PML technique. On the other hand, another benefit of our proposed PML method is that there is no need of fitting any non-physical parameter.

Notice also that in the continuous problem, before applying a discretization, the PML technique does not produce any reflection on the interface with the physical domain. However, after the discretization procedure, even using a singular absorbing function, the "perfectly matched" feature of the PML model is lost and numerical spurious reflected waves are generated on the interface boundary between the physical and PML domains.

\section{Further numerical simulations}

In this section we include some numerical simulations, which are not supported by any theoretical results ensuring the accuracy of the PML technique.

More precisely, in the next three subsections we describe, first, the classical Young's double-slit experiment, involving a scattering problem for the Helmholtz equation, stated in a unbounded domain included in a half-plane; second, the reliability test of a metallic component where one of its parts is several orders of magnitude larger than the rest, and so it can be modeled as an elasticity problem stated in an unbounded strip domain; and third, the computation of the scattering field generated by a semi-buried object in underwater sediments.

\subsection{Young's double-slit experiment}

This first numerical simulation resembles the well-known double-slit interference experiment stated originally by Thomas Young in 1801 to infer the wave-like nature of light. This model mimics the plane-wave excitation with two waveguides leading to slits in a screen and computes the diffraction pattern in a domain surrounding the apertures.

To compute the approximate solution, we consider a finite element method and a PML technique using the optimal absorbing function, as it is described in Subsection 6.2. We have used the domain shown in Figure 18 and a refinement of the mesh 
plotted in the same figure, with 51424 triangles and 6656 rectangles. We have taken as acoustic speed $c=340 \mathrm{~m} / \mathrm{s}$ and $\omega=272 \pi \mathrm{rad} / \mathrm{s}$.

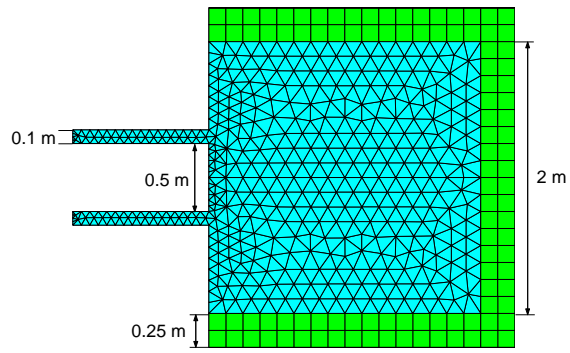

Fig. 18 Domain and coarse mesh for the double-slit interference test.

The left-hand side plot of Figure 19 shows the real part of the diffracted field near the double-slit.
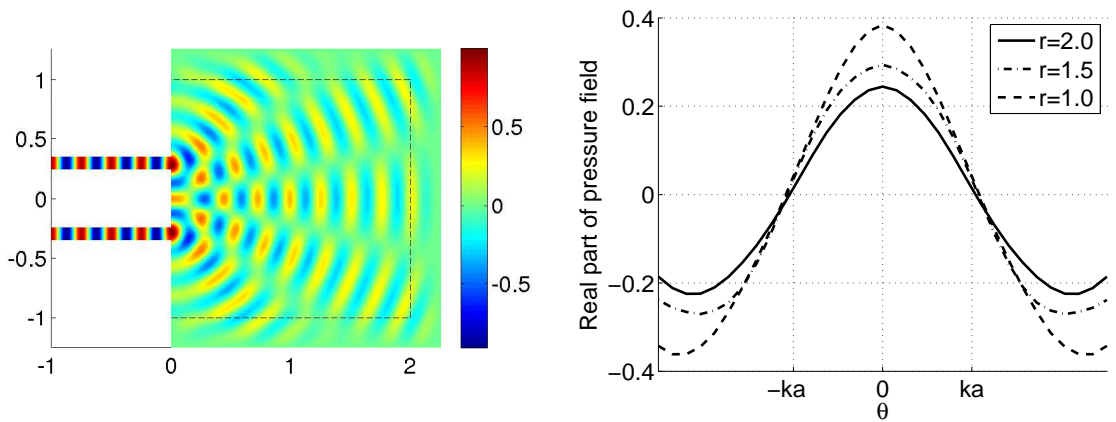

Fig. 19 Real part of the diffracted field generated by the excitation of the waveguides (left) and the interference pattern at different distances $r$ from the waveguides apertures (right).

When the Fresnel number is small enough, classical theory of Fraunhofer diffraction predicts a phenomenon of destructive interference which depends only on the angular coordinate $\theta$ (see for instance [21]). The interference lines are $\theta= \pm k a$, being $k=\omega / c$ the wavenumber and $a$ the radius of the aperture in the slits. It can be checked, in the left-hand side plot of Figure 19, that the numerical results achieve a good accuracy with respect to the approximate classical theory.

In the case of this problem, which is stated in a half-plane, a modification of the theoretical arguments shown in [24] allows us to prove that the use of a non-integrable absorbing function implies the exactness of the PML technique. In spite of the fact that the Green's function associated with the Helmholtz equation in a half-plane is analytic and known in a closed form, this theoretical modification is far from being obvious. 
7.2 Reliability test of a metallic component

Let us consider the two-dimensional vertical section of a metallic component, where the right part continues straight with the same transverse shape, and it is some orders of magnitude larger than the region depicted in Figure 20.

Our aim is to simulate numerically a $\mathrm{P}$-wave propagating from the left side through the rest of the device. Due to the difference of scale between the left and right parts, we consider that the computational domain is unbounded.

In the framework of the plane strain elasticity, the linear Hooke model is described by three physical parameters of the material: Young's modulus $E=2.7 \times 10^{9} \mathrm{~Pa}$, Poisson's ratio $\nu=0.3$ and mass density $\rho=7.86 \times 10^{3} \mathrm{Kg} / \mathrm{m}^{3}$.

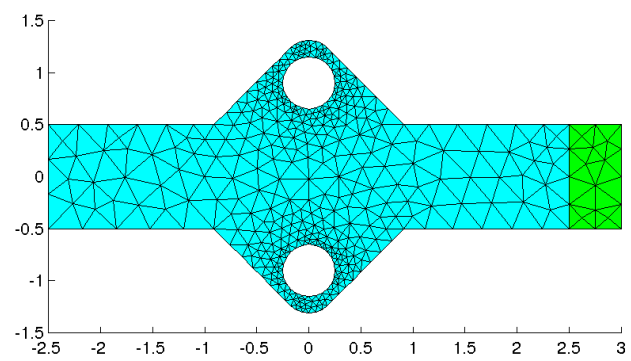

Fig. 20 Two-dimensional vertical section of the metallic component: the right part, which is assumed unbounded, is replaced by the PML domain (colored in green).

We assume null forces on the upper and lower boundaries and that the metallic component is fixed on the inner circumferences. To impose a $\mathrm{P}$-wave with unit amplitude incoming through the left boundary, we introduce the boundary condition

$$
(\Sigma(\mathbf{u}) \mathbf{n})_{j}-i \omega \sqrt{\frac{\rho E(1-\nu)}{(1+\nu)(1-2 \nu)}} u_{j}=2 i \omega \sqrt{\frac{\rho E(1-\nu)}{(1+\nu)(1-2 \nu)}} \quad j=1,2 .
$$

In order to assess how appropriate the use of the PML technique is, we compare naively the numerical solution obtained with the PML technique with the one computed if no special treatment is applied on the right boundary, but the computational domain is enlarged up to $x_{1}=12.5$.

We use a standard finite element method based on piecewise linear basis functions on a refined triangular mesh obtained from the coarse one depicted in Figure 20. It has been ensured that every edge element of the mesh is smaller than $\lambda / 15$ being $\lambda$ the wavelength of the $\mathrm{P}$-waves.

On one hand, if we use a Cartesian PML technique with absorbing functions $\gamma_{1}=\exp (i \pi / 6)$ and $\gamma_{2}=1$, in $\Omega_{\mathrm{A}}=(2.5,3) \times(-0.5,0.5)$, and in an enlarged PML domain $\Omega_{\mathrm{A}}=(6,7.5) \times(-0.5,0.5)$, the $\mathrm{L}^{2}$-relative difference between both solutions is $6.2 \%$, what illustrate the accuracy of the numerical approximation by using the PML technique in the small domain (see the middle and bottom plots in Figure 21). On the other hand, if we keep as reference solution that obtained with the PML technique in the largest computational domain and use a clamped condition on the right boundary but far from the physical region of interest, the relative difference increases up to 

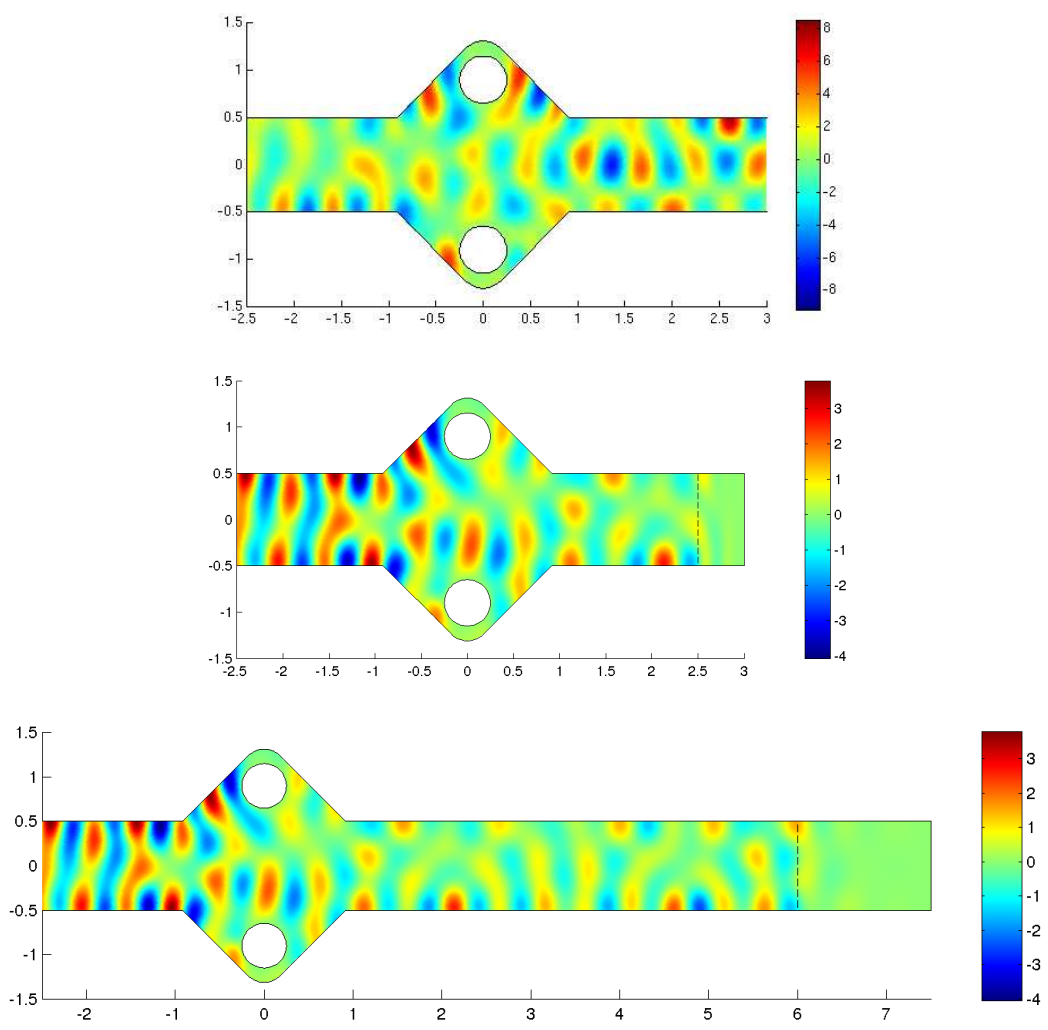

Fig. 21 Real part of the second component of the displacement $\mathbf{u}$ computed assuming a rigid wall termination on the right-hand side (upper), with a PML placed in $(2.5,3.0) \times(-0.5,0.5)$ (middle), and with a PML placed in $(6,7) \times(-0.5,0.5)$ (lower).

$78 \%$ even when the right boundary is imposed at the edge placed on $x_{1}=12.5$ (see the upper plot in Figure 21 where only the displacement has been plotted between $x_{1}=-2.5$ and $x_{1}=3.5$ ). Obviously, after the arguments described through this work these differences between the simulations were expected.

The mathematical analysis of the PML technique in this context is related to the study of wave propagation in elastic waveguides and it goes far from the scope of this work. Moreover, even in the framework of linear isotropic elasticity, the numerical behavior of the PML technique for elastic waveguides has not been studied until recently (see [75]).

\subsection{Semi-buried rigid object in underwater sediments}

Until now, we have dealt with different problems stated in unbounded domains, but homogeneous isotropic media have been always assumed in all the numerical simulations. 
Nevertheless, there exist several real-life applications, which involve stratified media, where the interfaces among layers are unbounded. Although the Green's function is not known in closed form, we can claim that the Cartesian PML technique is applicable with accurate numerical results.

Let us consider a rigid obstacle placed between two homogeneous layers as depicted in Figure 22. It is assumed that the top layer is filled with an isotropic acoustic fluid (water) with mass density $1000 \mathrm{~kg} / \mathrm{m}^{3}$ and sound speed $c_{1}=1500 \mathrm{~m} / \mathrm{s}$. The bottom layer involves a sand sediment governed by the Darcy's like porous model (already introduced Section 2) where the mass density is $1800 \mathrm{~kg} / \mathrm{m}^{3}$, the sound speed $c_{2}=$ $1600 \mathrm{~m} / \mathrm{s}$ and the flow resistivity $10000 \mathrm{~kg} /\left(\mathrm{m}^{3} \mathrm{~s}\right.$ ) (see [80] for other physical data).

In this case, the goal is focused in the numerical computation of the reflected pressure field generated by the scattering of an oblique plane wave of amplitude one and angle of incidence $2 \pi / 3$.

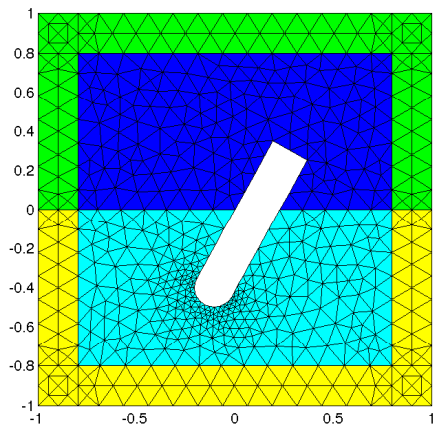

Fig. 22 Stratified domain composed by a fluid (water in blue) and a Darcy's like porous medium (sand sediment in cyan). The interface between both media continues straight through the PML domain.

Again a standard finite element method based on triangular meshes and piecewise linear basis functions has been used. The Cartesian PML setting involves a PML domain of thickness $0.2 \mathrm{~m}$ and constant absorbing functions, $\gamma_{1}=\gamma_{2}=\exp (i \pi / 6)$.

We have used a uniform refined mesh from the coarse one depicted in Figure 22. More precisely, a refined mesh satisfying that every edge is smaller that $\lambda / 15$, being $\lambda=\omega / c_{1}$ the wavelength associated with the time-harmonic motion in the top layer.

The real part of the reflected field is shown in Figure 23 for $\omega=9000 \pi \mathrm{rad} / \mathrm{s}$, where two different computational domains have been used. Notice that, in both cases, the thickness of the PML remains equal to $0.2 \mathrm{~m}$.

We have compared the solutions computed using the small and large domains and the $\mathrm{L}^{2}$-relative difference between them is $4.78 \%$. As far as the knowledge of the authors goes, there does not exist any theoretical result that guarantees the accuracy of numerical approximation in problems where the interface between two different media continues through the PML domain. Even in the simplest case where both media are homogeneous and isotropic, the problem remains open.

However, in the literature there exists a wide range of works dealing with stratified media, for instance, among many other areas, in geophysics (see [26]) or in underwater 

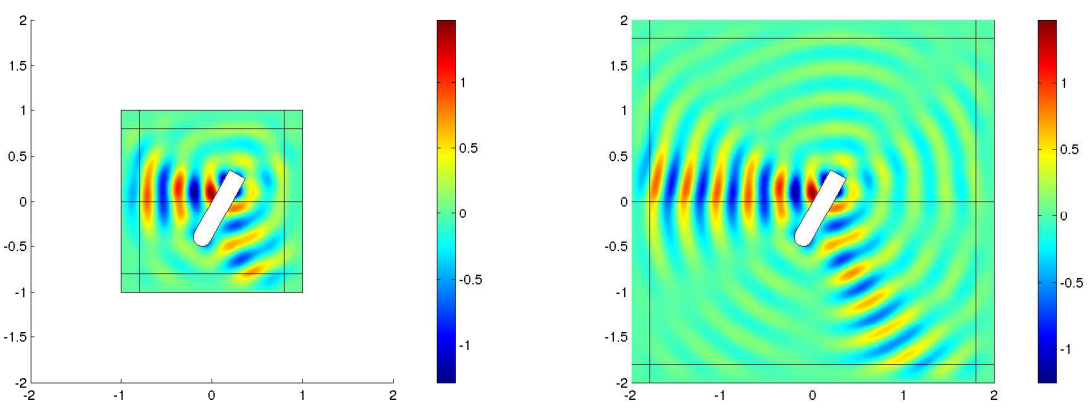

Fig. 23 Real part of the reflected field computed with a PML placed outside the box $(0.8,0.8) \times(0.8,0.8)($ left $)$, and with a PML placed in $(1.8,1.8) \times(1.8,1.8)$ (right), whose thickness is $0.2 \mathrm{~m}$ in both cases.

acoustics (see [53]), all of them with a common assumed claim: if the interface among the different media is perpendicular to the interface between the physical domain and the PML and continuous straight in the PML domain, then a good numerical accuracy is expected from the PML technique. To the best knowledge of the authors, this claims has not theoretical support yet.

This claimed numerical feature can be checked by comparing the same numerical simulation made in a larger domain, as we have done above. However, it is remarkable and challenging that this kind of reasonable behavior shown by the numerical results has not been analyzed yet for fluid-structure problems if the coupling interface is unbounded.

\section{Conclusions and further research}

We have revised the construction of the PML technique from different perspectives. First, it has been introduced from a physical point of view as a remedy for the drawbacks found in the Darcy's like porous model. Then, it has been illustrated one of the fundamental lacks of the PML technique: it is not possible setting a universal absorbing function which allows us to obtain always accurate results. In spite of this lack, it is possible to describe generally the PML technique for second order partial differential equations and obtain an existence and uniqueness result for elliptic problems.

Moreover, most of the contributions about this topic are related to the settling of optimal conditions for the absorbing functions in the PML domain. In the particular case of the Helmholtz equation, the optimality of the absorbing functions with non-integrable imaginary part has been illustrated numerically for the Cartesian PML technique and shown theoretically its exactness in the radial PML case. Notice that the numerical analysis of the optimal conditions, to minimize the numerical errors arising from the discretized PML problem, has not been provided yet.

As it has been sketched in the previous section, the mathematical analysis of the PML technique is far to be completed, even for mechanical problems where it is a usual numerical tool. We must mention some future computational challenges such as fluid/structure problems stated in unbounded domains, where the elastic solid and the 
coupling interfaces between fluid and solid domains are unbounded, and so they have to be included inside the PML domain. Even if the implementation of this problem in a computer code has not any technical difficulty, the mathematical analysis to derive existence and uniqueness, and the study of error estimates or the design of well-posedness absorbing functions is quite incomplete.

Despite time dependent problems are not included in the scope of this work, let us write some words. So basic problems as obtaining decay energy estimates for the time domain PML equations (even for standard choices of bounded absorbing functions) remain open. Obviously, the use of time-harmonic non-integrable absorbing functions for time dependent problems as the two-dimensional wave equation could also yield to optimal results. In fact, the extension of such optimality (as already discussed in Section 5) to time dependent problems is as difficult as the derivation of energy estimates in time-harmonic problems. In both cases, we need to obtain lower and upper bounds for the norm of the solution explicitly given with respect to the angular frequency.

Acknowledgements This work has been partially supported by Ministerio de Ciencia e Innovación of Spain under grant number MTM2008-02483. Moreover, the first and third author were partially supported by Xunta de Galicia under grant number PGIDIT-07-PXIB105257PR. The fourth author was partially supported by FONDAP and BASAL projects CMM, Universidad de Chile (Chile)

\section{References}

1. Abarbanel, S., Gottlieb, D.: On the construction and analysis of absorbing layers in CEM. Applied Numerical Mathematics 27(4), 331-340 (1998)

2. Abarbanel, S., Gottlieb, D., Hesthaven, J.S.: Well-posed perfectly matched layers for advective acoustics. J. Comp. Phys. 154(2), 266-283 (1999)

3. Aguilar, J., Combes, J.: A class of analytic perturbations for one-body Schrödinger Hamiltonians. Commun. Math. Phys. 22(4), 269-279 (1971)

4. Allard, J.F.: Propagation of sound in porous media: modelling sound absorbing materials. Elsevier, New York (1993)

5. Astley, R.J.: Infinite elements for wave problems: A review of current formulations and an assessment of accuracy. Int. J. Numer. Methods Eng. 49, 951-976 (2000)

6. Asvadurov, S., Druskin, V., Guddati, M.N., Knizhnerman, L.: On optimal finite-difference approximation of PML. SIAM J. Numer. Anal. 41(1), 287-305 (2004)

7. Bao, G., Wu, H.: Convergence analysis of the perfectly matched layer problems for timeharmonic Maxwell's equations. SIAM journal on numerical analysis 43(5), 2121-2143 (2006)

8. Basu, U., Chopra, A.K.: Perfectly matched layers for time-harmonic elastodynamics of unbounded domains: theory and finite-element implementation. Comput. Methods Appl. Mech. Engrg. 192(11), 1337-1375 (2003)

9. Bayliss, A., Turkel, E.: Radiation boundary conditions for wave-like equations. Comm. Pure Appl. Math. 33, 707-725 (1980)

10. Bécache, E., Bonnet-Benn Dhia, A.S., Legendre, G.: Perfectly matched layers for the convected Helmholtz equation. SIAM J. Numer. Anal. 42(1), 409-433 (2004)

11. Bécache, E., Joly, P.: On the analysis of Berenger's perfectly matched layers for Maxwell's equations. M2AN Math. Model. Numer. Anal. 36(1), 87-119 (2002)

12. Beranek, L.L., Vér, I.L.: Noise and Vibration Control Engineering: Principles and Applications. Wiley-Interscience (1992)

13. Bérenger, J.P.: A perfectly matched layer for the absortion of electromagnetics waves. J. Comp. Phys. 114(1), 185-200 (1994)

14. Bérenger, J.P.: Perfectly matched layer for the FDTD solution of wave-structure interaction problems. IEEE Trans. Antennas Propag. 44(1), 110-117 (1996)

15. Bérenger, J.P.: Three-dimensional perfectly matched layer for the absorption of electromagnetic waves. J. Comput. Phys. 127(2), 363-379 (1996) 
16. Bermúdez, A., Hervella-Nieto, L., Prieto, A., Rodríguez, R.: An exact bounded PML for the Helmholtz equation. C. R. Math. Acad. Sci. Paris 339(11), 803-808 (2004)

17. Bermúdez, A., Hervella-Nieto, L., Prieto, A., Rodríguez, R.: An optimal perfectly matched layer with unbounded absorbing function for time-harmonic acoustic scattering problems. J. Comput. Phys. 223, 469-488 (2007)

18. Bermúdez, A., Hervella-Nieto, L., Prieto, A., Rodríguez, R.: Validation of acoustic models for time harmonic dissipative scattering problems. J. Comput. Acous. 15(1), 95-121 (2007)

19. Bermúdez, A., Hervella-Nieto, L., Prieto, A., Rodríguez, R.: An exact bounded perfectly matched layer for time-harmonic scattering problems. SIAM J. Sci. Comput. 30(1), 312$338(2007 / 08)$

20. Bonet Ben-Dhia, A., Goursaud, B., Hazard, C., Prieto, A.: Finite element computation of leaky modes in stratified waveguides. In: A. Leger, M. Deschamps (eds.) Ultrasonic Wave Propagation in Non Homogeneous Media, vol. 128, pp. 73-86. Springer Proceedings in Physics, Springer Verlag (2008)

21. Born, M., Wolf, E.: Principles of Optics. Pergamon Press (1980)

22. Bramble, J.H., Pasciak, J.E.: Analysis of a finite PML approximation for the three dimensional time-harmonic Maxwell and acoustic scattering problems. Math. Comp. 76, 597-614 (2007)

23. Brekhovskikh, L.M., Godin, O.: Acoustics of Layered Media I: Plane and Quasi-Plane Waves. Springer (1999)

24. Chandler-Wilde, S.N., Monk, P.: The PML for rough surface scattering. Appl. Numer. Math. 59(9), 2131-2154 (2009)

25. Chew, W.C., Weedon, W.H.: A 3D perfectly matched medium from modified Maxwell's equations with stretched coordinates. IEEE Microwave Opt. Technol. Lett. 7(13), 599-604 (1994)

26. Cohen, G., Fauqueux, S.: Mixed spectral finite elements for the linear elasticity system in unbounded domains. SIAM J. Sci. Comput. 26, 864-884 (2005)

27. Collino, F., Monk, P.: Optimizing the perfectly matched layer. Comput. Methods Appl. Mech. Engrg. 164(1), 157-171 (1998)

28. Collino, F., Monk, P.: The Perfectly Matched Layer in Curvilinear Coordinates. SIAM J. Sci. Comput. 19(6), 2061-2090 (1998)

29. Collino, F., Tsogka, C.: Application of the PML absorbing layer model to the linear elastodynamic problem in anisotropic heterogeneous media. Geophysics 66(1), 294-307 (2001)

30. Colton, D., Kress, R.: Inverse Acoustic and Electromagnetic Scattering Theory. Springer Verlag, Berlin Heidelberg (1992)

31. Cummer, S.A.: Perfectly matched layer behavior in negative refractive index materials. IEEE Trans. Antennas Propagat. 3, 172-175 (2004)

32. Demkowicz, L., Ihlenburg, F.: Analysis of a coupled finite-infinite element method for exterior Helmholtz problems. Numer. Math. 88(1), 43-73 (2001)

33. Engquist, B., Majda, A.: Absorbing boundary conditions for numerical simulation of waves. Math. Comput. 31, 629-651 (1977)

34. Ervedoza, S., Zuazua, E.: Perfectly matched layers in 1-d: Energy decay for continuous and semi-discrete waves. Numer. Math. 109(4), 597-634 (2008)

35. Feng, K.: Finite element method and natural boundary reduction. In: Proc. Int. Congress of Mathematicians, pp. 1439-1453 (1983)

36. Gedney, S.D.: An anisotropic perfectly matched layer-absorbing medium for thetruncation of FDTD lattices. IEEE Trans. Antennas Propagat. 44(12), 1630-1639 (1996)

37. Gedney, S.D.: An anisotropic PML absorbing media for the FDTD simulation of fields in lossy and dispersive media. Electromagnetics 16(4), 399-415 (1996)

38. Givoli, D.: Non-reflecting boundary conditions. J. Comput. Phys. 94(1), 1-29 (1991)

39. Givoli, D.: Numerical Methods for Problems in Infinite Domains. Elsevier (1992)

40. Givoli, D., Neta, B.: High-order non-reflecting boundary scheme for time-dependent waves. J. Comput. Phys. 186(1), 24-46 (2004)

41. Hagstrom, T.: New results on absorbing layers and radiation boundary conditions. In: M. Ainsworth, P. Davies, D. Duncan, P. Martin, B. Rynne (eds.) Topics in Computational Wave Propagation: Direct and Inverse Problems, pp. 1-39. Lecture Notes in Computational Science and Engineering, Springer Verlag (2003)

42. Hagstrom, T., Lau, S.: Radiation Boundary Conditions for Maxwells Equations: A Review of Accurate Time-domain Formulations. Journal of Computational Mathematics 25(3), 305-336 (2007) 
43. Harari, I., Albocher, U.: Studies of FE/PML for exterior problems of time-harmonic elastic waves. Comput. Methods Appl. Mech. Engrg. 195(29-32), 3854-3879 (2006)

44. Harari, I., Slavutin, M., Turkel, E.: Analytical and numerical studies of a finite element PML for the Helmholtz equation. J. Comput. Acoust 8(1), 121-137 (2000)

45. Hislop, P., Sigal, I.: Introduction to spectral theory with applications to Schrödinger operators. Series in Applied Mathematical Sciences, Springer (1996)

46. Hohage, T., Schmidt, F., Zschiedrich, L.: Solving time-harmonic scattering problems based on the pole condition. II: Convergence of the PML method. SIAM J. Math. Anal. 35(3), $547-560$ (2004)

47. Hu, F.Q.: A stable, perfectly matched layer for linearized euler equations in unsplit physical variables. J. Comput. Phys. 173(2), 455-480 (2001)

48. Ihlenburg, F.: Finite Element Analysis of Acoustic Scattering. Springer Verlag (1998)

49. Johnson, S., Bienstman, P., Skorobogatiy, M., Ibanescu, M., Lidorikis, E., Joannopoulos, J.: Adiabatic theorem and continuous coupled-mode theory for efficient taper transitions in photonic crystals. Phys. Rev. E 66, 066,608 (2002)

50. van Joolen, V.J., Neta, B., Givoli, D.: High-order Higdon-like boundary conditions for exterior transient wave problems. Int. J. Numer. Methods. Eng. 63(7), 1041-1068 (2005)

51. Kato, T.: Perturbation theory for linear operators. Springer (1995)

52. Kim, S., Pasciak, J.: Analysis of the spectrum of a cartesian perfectly matched layer (PML) approximation to acoustic scattering problems Preprint of the Department of Mathematics, Texas A\&M University

53. Kormann, J., Cobo, P., Prieto, A.: Perfectly matched layers for modelling seismic oceanography experiments. J. Sound Vibration 317(1-2), 354-365 (2008)

54. Kreiss, H.O., Lorenz, J.: Initial-Boundary Problems and the Navier-Stokes Equation. Academic Press, New York (1989)

55. Kuzuoglu, M., Mittra, R.: Frequency dependence of the constitutive parameters of causalperfectly matched anisotropic absorbers. IEEE Microw. Guided W. 6(12), 447-449 (1996)

56. Lassas, M., Liukkonen, J., Somersalo, E.: Complex Riemannian metric and absorbing boundary conditions. J. Math. Pures Appl 80(7), 739-768 (2001)

57. Lassas, M., Somersalo, E.: On the existence and convergence of the solution of PML equations. Computing 60(3), 229-242 (1998)

58. Lassas, M., Somersalo, E.: Analysis of the PML equations in general convex geometry. Proc. Roy. Soc. Edinburgh Sect. A 131(5), 1183-1207 (2001)

59. Lions, J.L., Métral, J., Vacus, O.: Well-posed absorbing layer for hyperbolic problems. Numer. Math. 92(3), 535-562 (2002)

60. Marcuse, D.: Theory of dielectric optical waveguides. Academic Press, Inc., New York (1974)

61. Masmoudi, M.: Numerical solution for exterior problems. Numer. Math. 51(1), 87-101 (1987)

62. Navon, I.M., Neta, B., Hussaini, M.Y.: A perfectly matched layer approach to the linearized shallow water equations models. Monthly Weather Review 132(6), 1369-1378 (2004)

63. Nédélec, J.C.: Acoustic and Electromagnetic Equations. Integral Representations for Harmonic Problems. Springer Verlag (2000)

64. Oskooi, A., Zhang, L., Avniel, Y., Johnson, S.: The failure of perfectly matched layers, and towards their redemption by adiabatic absorbers. Ops. Express 16(15), 11,376-11,392 (2008)

65. Petropoulos, P.G.: Reflectionless sponge layers as absorbing boundary conditions for the numerical solution of Maxwell equations in rectangular, cylindrical, and spherical coordinates. SIAM J. Appl. Math. 60(3), 1037-1058 (2000)

66. Prieto, A.: Some contributions in time-harmonic dissipative acoustic problems. Ph.D. thesis, Departamento de Matemática Aplicada, Universidade de Santiago de Compostela (2007)

67. Qi, Q., Geers, T.L.: Evaluation of the Perfectly Matched Layer for Computational Acoustics. J. Computat. Phys. 139(1), 166-183 (1998)

68. Rappaport, C.M.: Interpreting and Improving the PML Absorbing Boundary Condition Using Anisotropic Lossy Mapping of Space. IEEE Trans. Magn. 32(3), 968-974 (1996)

69. Roden, J.A., Gedney, S.D.: Convolutional PML (CPML): An efficient FDTD implementation of the CFS-PML for arbitrary media. Microw. Opt. Technol. Lett. 27(5), 334-338 (2000)

70. Rudin, W.: Real and complex analysis. Series in Higher Mathematics, McGraw-Hill (1974) 
71. Sacks, Z.S., Kingsland, D.M., Lee, R., Lee, J.F.: A perfectly matched anisotropic absorber for use as an absorbing boundary condition. IEEE Trans. Antennas Propagat. 43(12), 1460-1463 (1995)

72. Shirron, J.J., Babuska, I.: A comparison of approximate boundary conditions and infinite element methods for exterior Helmholtz problems. Comput. Methods Appl. Mech. Engrg. 164(1), 121-139 (1998)

73. Simon, B.: Resonances and complex scaling: A rigorous overview. Int. J. Quantum Chem. 14(4), 529-542 (1978)

74. Sjögreen, B., Petersson, N.A.: Perfectly matched layers for Maxwells equations in second order formulation. J. Comput. Phys. 209(1), 19-46 (2005)

75. Skelton, E.A., Adams, S.D.M., Craster, R.V.: Guided elastic waves and perfectly matched layers. Wave Motion 44(7-8), 573-592 (2007)

76. Teixeira, F.L., Chew, W.C.: Systematic derivation of anisotropic PML absorbing media in cylindrical and spherical coordinates. IEEE Microw. Guided Wave Lett. 7(11), 371-373 (1997)

77. Teixeira, F.L., Chew, W.C.: Extension of the PML absorbing boundary condition to 3D spherical coordinates: scalar case. IEEE Trans. Magn. 34(5 Part 1), 2680-2683 (1998)

78. Turkel, E., Yefet, A.: Absorbing PML boundary layers for wave-like equations. Appl. Numer. Math 27(4), 533-557 (1998)

79. Ying, L.A.: Numerical methods for exterior problems, Peking University, Series in Mathematics, vol. 2. World Scientific (2006)

80. Zampolli, M., Tesei, A., Jensen, F., Malm, N., Blottman, J.: A computationally efficient finite element model with perfectly matched layers applied to scattering from axially symmetric objects. J. Acoust. Soc. Am. 122(3), 1472-1485 (2007)

81. Zeng, Y.Q., He, J.Q., Liu, Q.H.: The application of the perfectly matched layer in numerical modeling of wave propagation in poroelastic media. Geophysics 66(4), 1258-1266 (2006)

82. Zhao, L., Cangellaris, A.: A general approach for the development of unsplit-field timedomain implementations of perfectly matched layers for FDTD gridtruncation. IEEE Microw. Guided Wave Lett. 6(5), 209-211 (1996)

83. Zschiedrich, L., Klose, R., Schädle, A., Schmidt, F.: A new finite element realization of the perfectly matched layer method for Helmholtz scattering problems on polygonal domains in two dimensions. J. Comput. Appl. Math. 188(1), 12-32 (2006) 